ARTICLE

DOI: $10.1038 /$ s41467-018-03638-6

\title{
The role of CSF1R-dependent macrophages in control of the intestinal stem-cell niche
}

\author{
Anuj Sehgal (1) ${ }^{1}$, David S. Donaldson ${ }^{1}$, Clare Pridans (10 ${ }^{1,2}$, Kristin A. Sauter ${ }^{1}$, David A. Hume ${ }^{1,2,3}$ \\ $\&$ Neil A. Mabbott (1) ${ }^{1}$
}

Colony-stimulating factor 1 (CSF1) controls the growth and differentiation of macrophages. CSF1R signaling has been implicated in the maintenance of the intestinal stem cell niche and differentiation of Paneth cells, but evidence of expression of CSF1R within the crypt is equivocal. Here we show that CSF1R-dependent macrophages influence intestinal epithelial differentiation and homeostasis. In the intestinal lamina propria CSF1R mRNA expression is restricted to macrophages which are intimately associated with the crypt epithelium, and is undetectable in Paneth cells. Macrophage ablation following CSF1R blockade affects Paneth cell differentiation and leads to a reduction of $\mathrm{Lgr}^{+}$intestinal stem cells. The disturbances to the crypt caused by macrophage depletion adversely affect the subsequent differentiation of intestinal epithelial cell lineages. Goblet cell density is enhanced, whereas the development of $M$ cells in Peyer's patches is impeded. We suggest that modification of the phenotype or abundance of macrophages in the gut wall alters the development of the intestinal epithelium and the ability to sample gut antigens.

\footnotetext{
${ }^{1}$ The Roslin Institute \& Royal (Dick) School of Veterinary Sciences, University of Edinburgh, Easter Bush, Midlothian EH25 9RG, UK. ${ }^{2}$ MRC Centre for Inflammation Research, University of Edinburgh, The Queen's Medical Research Institute, 47 Little France Crescent, Edinburgh EH16 4TJ, UK. ${ }^{3}$ Mater Research-University of Queensland, Translational Research Institute, Woolloongabba, QL 4102, Australia. These authors contributed equally: Anuj Sehgal, David S. Donaldson. Correspondence and requests for materials should be addressed to N.A.M. (email: neil.mabbott@roslin.ed.ac.uk)
} 
$\mathrm{M}$ ucosal surfaces such as those in the gastrointestinal and respiratory tracts are continuously exposed to commensal and pathogenic microorganisms. In the intestine a single layer of epithelial cells bound by tight-junctions limits the access of these microorganisms to the underlying host tissues. This epithelium is self-renewing and is continually replaced every 5-7 days. The crypts at the base of each intestinal villus contain populations of cycling leucine-rich repeat-containing $\mathrm{G}$ protein-coupled receptor 5 (Lgr5)-expressing intestinal stem cells ${ }^{1}$. These stem cells produce highly proliferative transit- amplifying daughter cells that, depending on the stimuli they receive, can differentiate into all the epithelial cell populations within the lining of the small intestine, including: enterocytes, goblet cells, enteroendocrine cells, tuft cells, and Paneth cells. Differentiated cell types, with the exception of the Paneth cells, migrate along the epithelium of the villus where they perform their physiological function before being subsequently lost via apoptosis as they reach the villus tip. Paneth cells remain located within the base of the crypts where they are interspersed between the Lgr $5^{+}$intestinal stem cells. Paneth cells release antimicrobial a

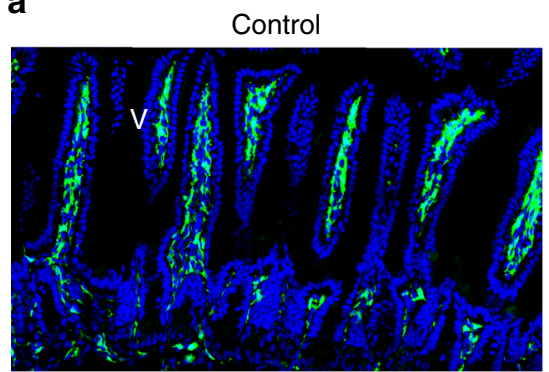

b

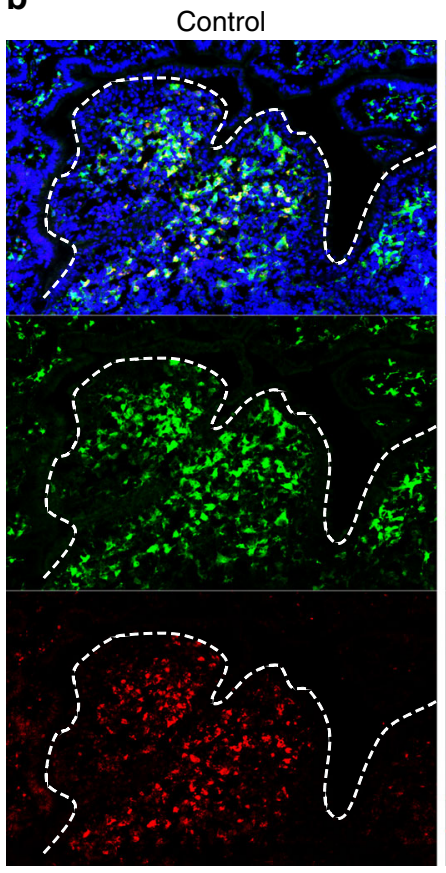

Anti-CSF1R

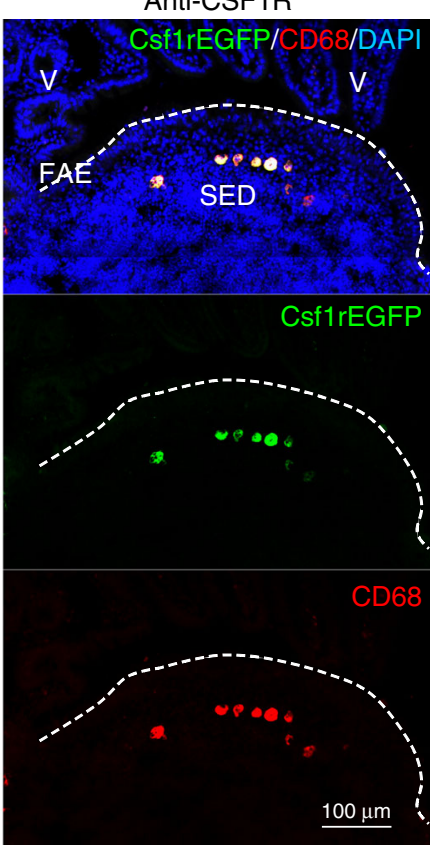

d

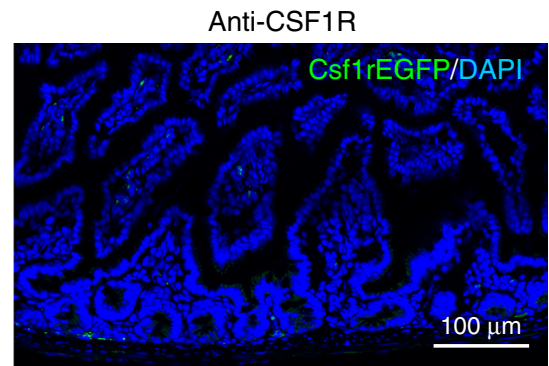

C
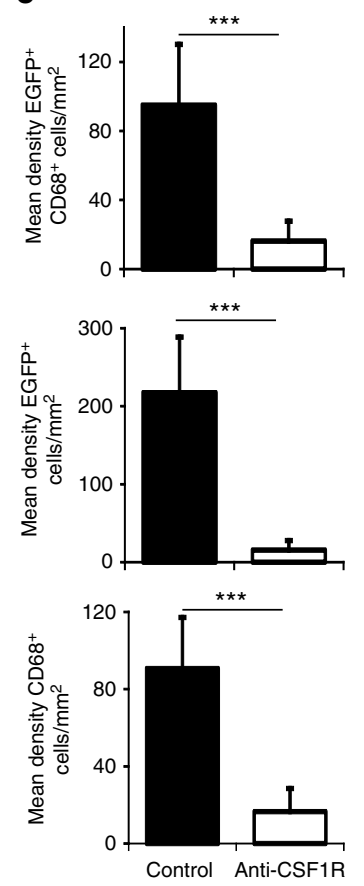

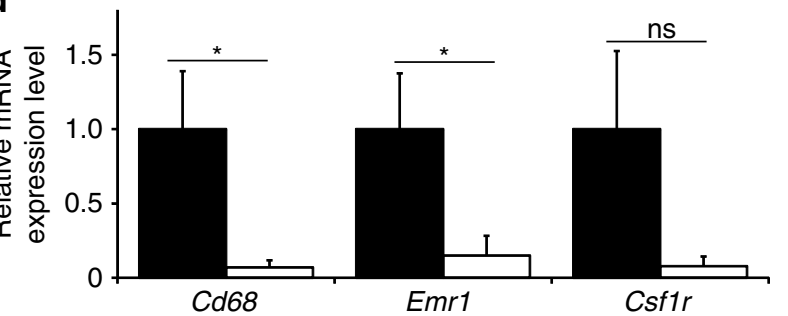

Fig. 1 Prolonged anti-CSF1R blockade depletes macrophages in the gut. Csf1r-EGFP mice were treated with anti-CSF1R mAb or control-lgG (control) for 6 weeks before analysis. a Depletion of Csf1r-EGFP+ (green) macrophages in the gut wall of anti-CSF1R mAb-treated mice. Scale bar, $100 \mu$ m. Images are representative of four mice/group from three independent experiments. b Detection of Csf1r-EGFP ${ }^{+}$(green) and CD68 ${ }^{+}$(red) macrophages in Peyer's patches by IHC. FAE, follicle-associated epithelium; SED, subepithelial dome; V, villus. Broken lines indicate the FAE boundary. Scale bar, $100 \mu$ m. c Morphometric analysis of the density of $\mathrm{Csffr}_{\mathrm{EGFP}}{ }^{+} \mathrm{CD} 68^{+}$macrophages in Peyer's patches. Data represent mean $\pm \mathrm{SD}$ from 2 to 10 sections from 3 to 4 mice/group. ${ }^{\star \star \star} P<0.001$, two-tailed unpaired Student's $t$-test. d RT-qPCR analysis of Cd68, Emr1, and Csf1r expression in Peyer's patches. Bars represent mean \pm SEM. Data are derived from 3 to 4 mice/group. ${ }^{\star} P<0.05$, two-tailed unpaired Student's $t$-test. NS not significant 
a

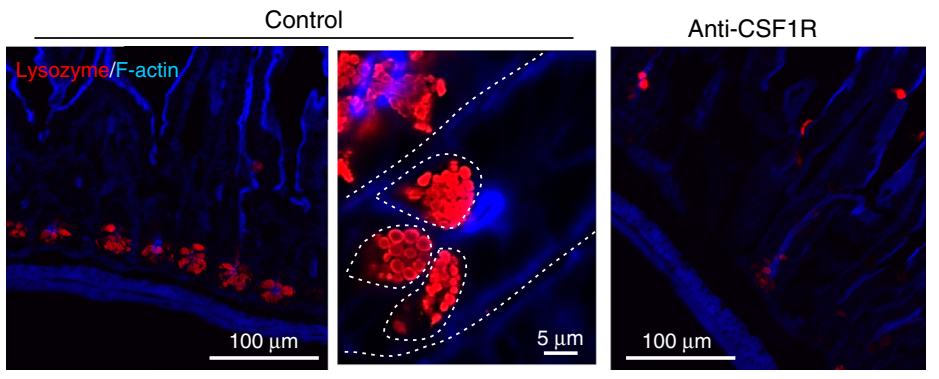

b

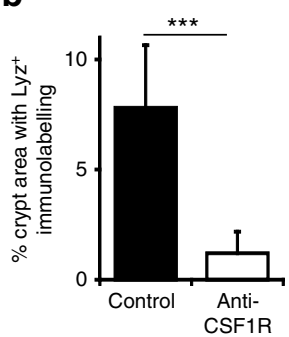

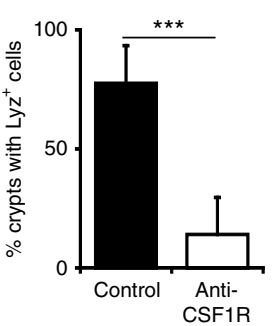

C

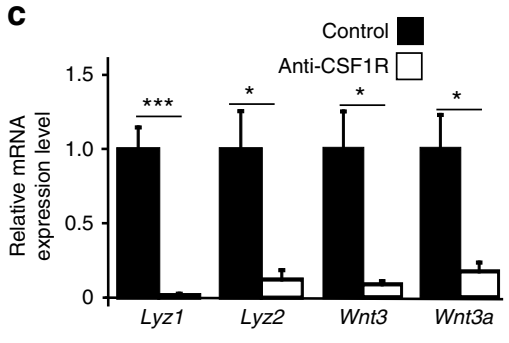

d

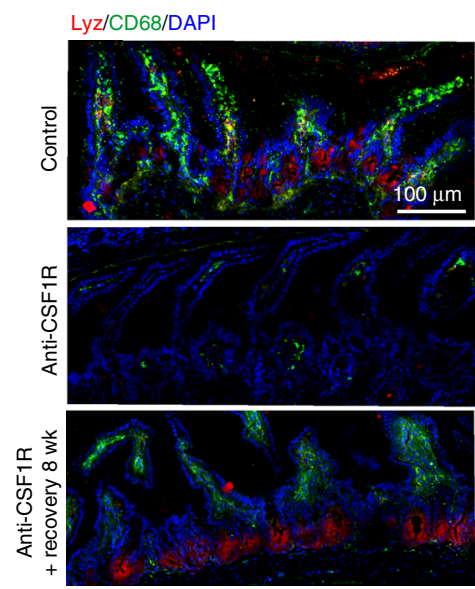

e

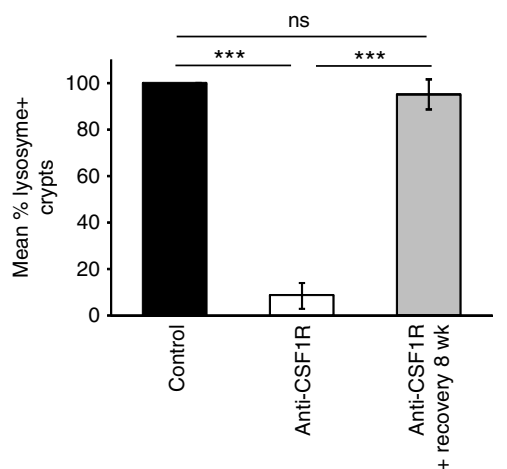

Fig. 2 Prolonged anti-CSF1R blockade leads to a loss of lysozyme-expressing Paneth cells in the crypts. Mice were treated with anti-CSF1R mAb or controlIgG (control) for 6 weeks before subsequent analysis. a IHC analysis shows loss lysozyme-expressing Paneth cells (red) in intestinal crypts after antiCSF1R-treatment. Broken line indicates crypt and Paneth cell boundaries. Images are representative of 4 mice/group from 3 independent experiments. $\mathbf{b}$ Morphometric analysis of the density of lysozyme-expressing Paneth cells in the crypts after anti-CSF1R mAb treatment (open bars) when compared to controls (closed bars). Data represent mean \pm SD from 6 to 20 sections from four mice/group, and are representative of data derived from three independent experiments. ${ }^{\star \star \star} P<0.001$, Mann-Whitney U-test. c RT-qPCR analysis shows negligible expression of Lyz1, Lyz2, Wnt3, and Wnt3a in mRNA from isolated intestinal crypts following treatment with anti-CSF1R mAb. Data are normalized to the expression of Gapdh. Bars represent

mean \pm SEM. Data are derived from four mice/group. ${ }^{\star} P<0.05$; ${ }^{\star \star \star} P<0.001$, two-tailed unpaired Student's $t$-test. d Mice were treated with anti-CSF1R $\mathrm{mAb}$ or control-lgG (control) for 6 weeks. A parallel group of mice were allowed to recover for 8 week following anti-CSF1R mAb. IHC analysis shows loss lysozyme-expressing Paneth cells (red) in intestinal crypts and $\mathrm{CD}_{6} 8^{+}$macrophages (green) in the gut wall after anti-CSF1R-treatment. The presence of these cells was restored within an 8 week recovery period following anti-CSF1R-treatment. Scale bar $100 \mu$ m. e Morphometric analysis of the \% crypts with lysozyme-expressing Paneth cells mice from each treatment group. Data represent mean \pm SD from sections from 3 to 4 mice/group, and 30 to 120 crypts/section, and are representative of data from three independent experiments. ${ }^{\star \star \star} P<0.001$, one-way ANOVA with Tukey's post hoc test. NS not significant

products such as lysozyme and alpha defensins which help to protect the crypt from bacterial infection, alongside homeostatic factors such as Wnt-signaling molecules which are essential for the maintenance of the $\operatorname{Lgr} 5^{+}$intestinal stem cells. Accordingly, when Paneth cells are specifically depleted there is a concomitant reduction in $\operatorname{Lgr}^{+}$stem cells ${ }^{2}$.

The lamina propria of the intestine contains an abundant population of macrophages that are renewed continuously from the circulating monocyte population ${ }^{3,4}$. Monocyte-macrophage differentiation is controlled by colony-stimulating factor (CSF1), and mice lacking this factor $(o p / o p)$ or the receptor, CSF1R, are profoundly macrophage deficient ${ }^{5}$. CSF1 signaling is also required for maintenance of the macrophage populations in adult mice. Treatment of adult mice with a blocking anti-CSF1R antibody produces an almost complete depletion of tissue macrophage populations in most organs, including the intestinal lamina propria ${ }^{6}$. Conversely, treatment of mice with $\mathrm{CSF}^{7}$, or with a recently-developed recombinant CSF1-Fc conjugate, induces extensive macrophage infiltration into tissues ${ }^{8}$.

The $o p / o p$ mouse also lacks lysozyme-expressing Paneth cells, and shows a commensurate reduction in Lgr5 expression and cell proliferation in the $\mathrm{crypt}^{9,10}$. Immunostaining with an anti- 
CSF1R antiserum suggested that the protein was expressed by Paneth cells implying that CSF1 directly regulates their development $^{9-11}$. By contrast, a Csf1r-EGFP reporter mouse, in which the Csf1r promoter drives the expression of EGFP ${ }^{12}$, labels tissue macrophages but not the Paneth cells, or indeed any epithelial cell lineage throughout the lining of the small intestine ${ }^{13}$.

CSF1-dependent macrophages exhibit many important roles in the maintenance of tissue homeostasis and repair ${ }^{14}$. For example, the macrophages in the muscularis externa of the wall of the gut can respond to luminal bacterial infections, produce bone morphogenetic protein 2 and interact with enteric neurons to regulate gastrointestinal motility ${ }^{15,16}$. The neurons in turn, produce CSF1. Therefore, in the current study, we tested the hypothesis that the effect of CSF1R blockade on the maintenance of Paneth cells in the intestinal crypts was indirect. Indeed, we show here that CSF1R-dependent macrophages are essential for the constitutive homeostatic maintenance of the intestinal crypt.

In gut-associated lymphoid tissues (GALT), Lgr5 ${ }^{+}$intestinal stem cells within the dome-associated crypts also give rise to $M$ cells ${ }^{17}$. These unique epithelial cells are specialized for the a

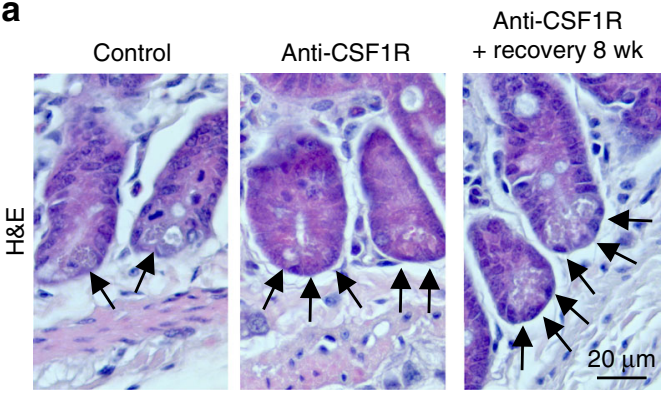

C

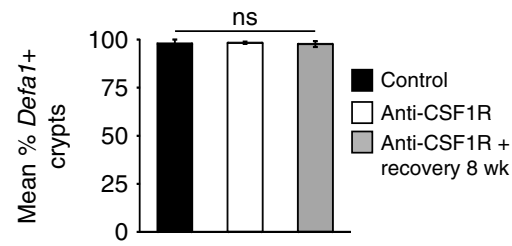

d
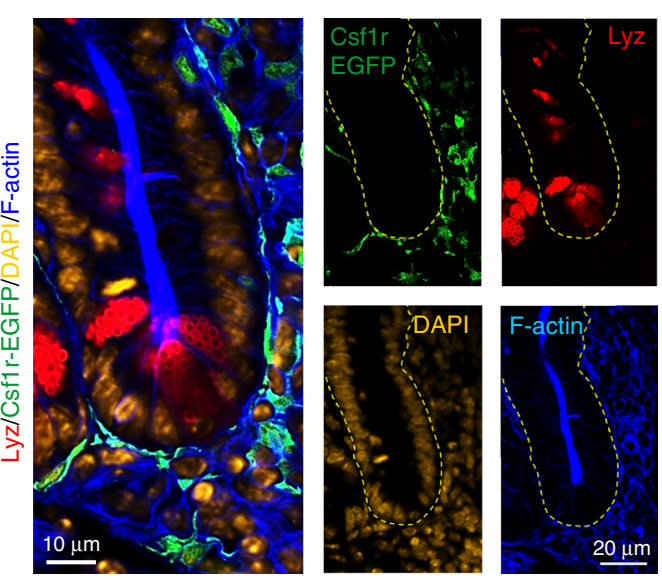

f

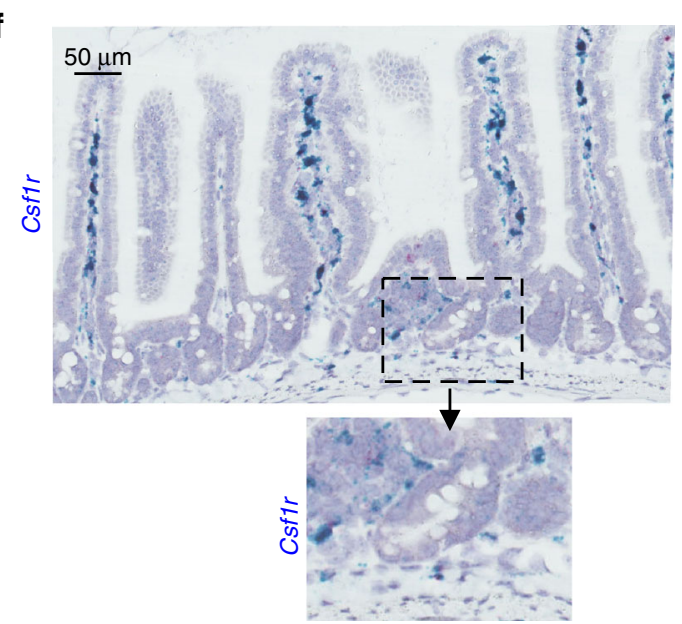

b
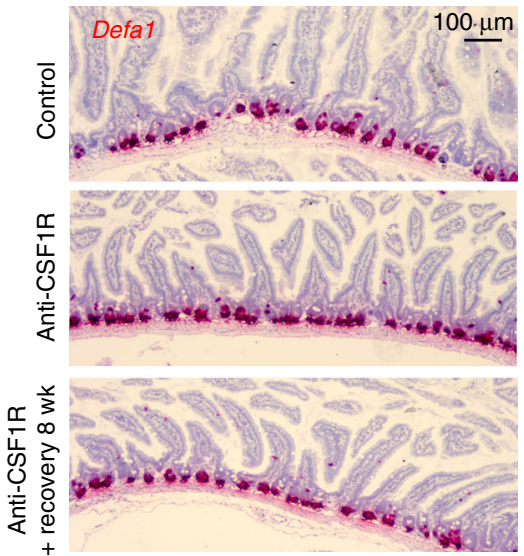

e

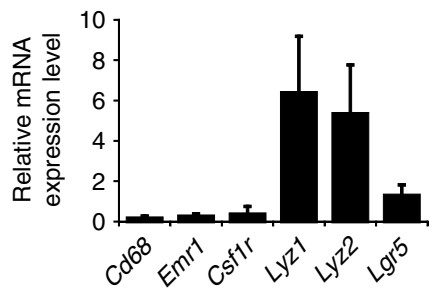

g
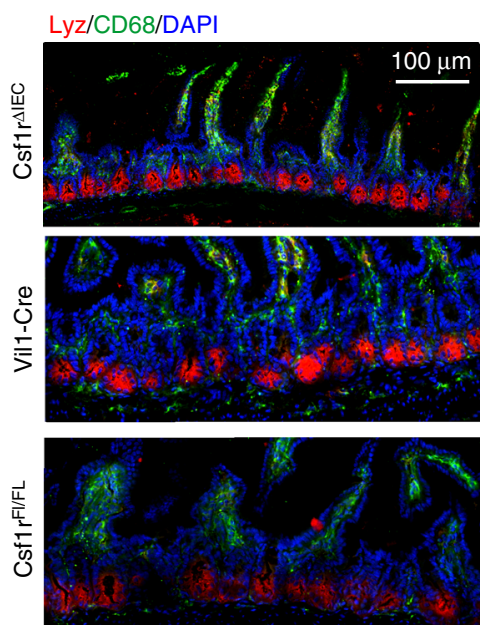

h

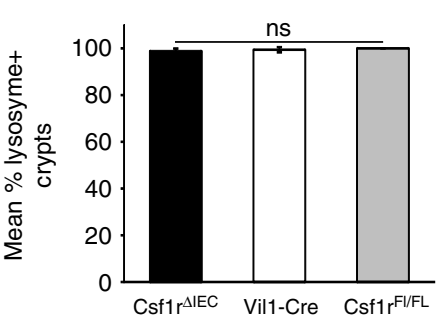


transcytosis of lumenal particulate antigens and pathogens across the follicle-associated epithelium $(\mathrm{FAE})^{18}$. The transcytosis of particulate antigens from the gut lumen by $M$ cells is an important first step in the induction of an efficient mucosal immune response ${ }^{19-21}$. Since $\mathrm{Lgr}^{+}$intestinal stem cells are adversely affected in absence of Paneth cells ${ }^{2}$ or CSF1R signaling ${ }^{9,10}$, we also tested the hypothesis that prolonged CSF1R blockade indirectly affects the functional differentiation of $\mathrm{M}$ cells. A link between macrophage function and antigen sampling provides an obvious mechanism to ensure that antigens derived from the gut are recognized by the innate immune system.

In this study, we show that CSF1R mRNA expression is undetectable in Paneth cells within intestinal crypts and is instead restricted to macrophages which are intimately associated with the crypt epithelium. The depletion of these macrophages following prolonged CSF1R blockade disturbs intestinal crypt homeostasis, affecting the differentiation of Paneth cells and Lgr $5^{+}$intestinal stem cells. The disturbances to the crypt caused by macrophage depletion adversely affect the subsequent differentiation of intestinal epithelial cell lineages, changing the balance between goblet cell and M-cell differentiation. Taken together, our observations reveal that CSF1R-dependent crypt-associated macrophages are constitutively required to maintain the intestinal stem-cell niche in the small intestine. This suggests that modification of the phenotype or abundance of macrophages in the gut wall, for example after pathogen infection, could adversely affect the development of the intestinal epithelium and the ability of the mucosal immune system to sample particulate antigens from the gut lumen.

\section{Results}

Prolonged CSF1R blockade depletes macrophages throughout the gut. Prolonged CSF1R blockade was achieved by treatment of C57BL/6J wild-type mice or Csf1r-EGFP mice with anti-CSF1R mAb M279 (or normal rat IgG as a control) three times a week for a 6 week period ${ }^{13}$. The efficacy of the CSF1R blockade was confirmed by the $>95 \%$ reduction of Csf1r-EGFP ${ }^{+}$macrophages throughout the wall of the gut (Fig. 1a), in Peyer's patches (Fig. 1b, c) and bone marrow (Supplementary Fig. 1). The expression of Csflr and typical macrophage-specific transcripts including Cd68 and Emr1 (also known as Adgre1, encoding the F4/80 antigen) was also reduced in mRNA from the ileum of antiCSF1R-treated mice (Fig. 1d).

Constitutive CSF1R stimulation maintains Paneth cell maturation. Previous studies have shown that the status of the intestinal crypts is disturbed in mice lacking CSF1R signaling ${ }^{9-11}$. The number and density of lysozyme-expressing Paneth cells within the intestinal crypts was significantly reduced after prolonged CSF1R blockade (Fig. 2a, b). A similar reduction in lysozyme mRNA expression, as well as the Paneth cell-derived homeostatic factors Wnt3 and Wnt $3 a$ was observed in mRNA from crypts isolated from the intestines of anti-CSF1R mAbtreated mice (Fig. 2c). The effects of CSF1R blockade on Paneth cell status were transient. Lysozyme expression in Paneth cells in intestinal crypts was restored to the same levels as control-treated mice when the mice were allowed to recover for $8 \mathrm{wk}$ following anti-CSF1R mAb treatment (Fig. 2d, e). Prolonged CSF1R blockade did not, in fact, lead to the depletion of Paneth cells. Cells containing abundant cytoplasmic secretory granules clearly remained in the intestinal crypts of mice treated with anti-CSF1R $\mathrm{mAb}$ (Fig. 3a). Paneth cells characteristically secrete a large range of antimicrobial factors including alpha defensins. RNA in situ hybridization analyses indicated that Defal (encoding alphadefensin 1) mRNA was still abundant in the intestinal crypts of mice treated with anti-CSF1R mAb (Fig. 3b). Furthermore, after prolonged CSF1R blockade the number of crypts with Defal mRNA-expressing Paneth cells was similar to those observed in the intestines of control-treated mice (Fig. 3b, c). Taken together, these data clearly indicate that CSF1R signaling is not required for Paneth cell survival, but instead, controls their differentiation.

Paneth cells do not express CSF1R. The CSF1R dependence of Paneth cells has been attributed to the expression of CSF1R in these cells $s^{9,10}$, but could also reflect an indirect requirement for macrophages in intestinal development. For example, CSF1deficient mice lack insulin-secreting beta cells in the pancreas ${ }^{22}$, but these cells were not depleted in ant-CSF1R treated mice ${ }^{13}$. Using confocal imaging we examined the expression of CSF1R in the intestines of the Csf1r-EGFP reporter "MacGreen" mouse in which the Csf1r promoter drives the expression of $\mathrm{EGFP}^{12}$. In these mice, the only additional sites of EGFP expression are placental trophoblasts, accurately reflecting the expression of Csf1r mRNA in these cells from a distal, unique promoter ${ }^{12}$, and granulocytes which express Csf1r mRNA but do not translate CSF1R protein ${ }^{23}$. The expression of EGFP in these mice is highly stable and reliably identifies Csflr-expressing cells even in situations where Csflr mRNA is depleted, such as following the trans-differentiation of macrophages into smooth muscle-like cells $^{24}$. In the intestines of Csf1r-EGFP mice, EGFP expression was not detected in lysozyme-expressing Paneth cells in the intestinal crypts (Fig. 3d). As anticipated, no background fluorescence was observed in the intestines of non-transgenic C57BL/6 control mice (Supplementary Fig. 2). The absence of CSF1R expression in Paneth cells is further supported by the negligible expression of Csflr in mRNA from isolated intestinal crypts (Fig. 3e), and the absence of Csflr mRNA in data from independent mRNA sequencing studies of intestinal crypts and

Fig. 3 Paneth cells do not express CSF1R mRNA and protein. Mice were treated with anti-CSF1R mAb or control-IgG (control) for 6 weeks before analysis. A parallel group of mice were allowed to recover for 8 wk following anti-CSF1R mAb treatment. a Analysis of H\&E stained intestines revealed that Paneth cells containing secretory granules (arrows) were present following prolonged CSF1R blockade. Scale bar, $20 \mu \mathrm{m}$. Representative images from 3 mice/ group are shown. b RNA in situ hybridization analyses showed that Defa1 mRNA (red) was abundantly expressed in the intestinal crypts of mice treated with anti-CSF1R mAb. Scale bar, $100 \mu \mathrm{m}$. Representative images from 3 mice/group are shown. c Morphometric analysis of the \% crypts with Defa1expressing Paneth cells in the crypts of mice from each treatment group. Data represent mean \pm SD derived from 3 mice/group, and 96-100 crypts/ section. ns, not significant, one-way ANOVA with Tukey's post hoc test. d IHC analysis of the crypts of untreated Csf1r-EGFP mice shows Paneth cells do not express CSF1R. In the crypt EGFP expression was only detected in cells with a macrophage morphology. e RT-qPCR analysis shows negligible expression of Cd68, Emr1, and Csf1r in mRNA from isolated intestinal crypts. Data are normalized to the expression of Gapdh. $\mathbf{f}$ RNA in situ hybridization analyses showed that Csf1r mRNA (blue) was not expressed in the epithelium of the small intestine. Scale bar, $50 \mu$ m. Inset shows absence of Csf1r mRNA in the epithelium of the intestinal crypt. $\mathbf{g} \mathrm{IHC}$ analysis of lysozyme-expressing Paneth cells (red) in intestinal crypts and CD68 ${ }^{+}$macrophages (green) in the gut wall of Csf1r ${ }^{\Delta \mathrm{IEC}}$, Vil1-Cre and Csf1r FL/FL mice. Representative images from three mice/group are shown. Scale bar $100 \mu$ m. $\mathbf{h}$ Morphometric analysis revealed that the \% crypts with lysoszyme-expressing Paneth cells was similar in the intestines of Csf1r $\Delta \mathrm{IEC}, \mathrm{Vil1-Cre}$ and Csf1r FL/FL mice. Data represent mean \pm SD derived from three mice/group, and 34-118 crypts/section. NS not significant, one-way ANOVA with Tukey's post hoc test 
a
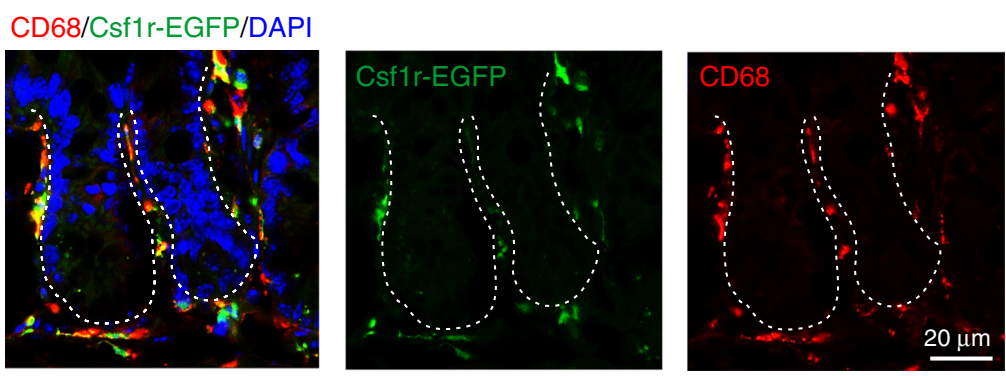

b
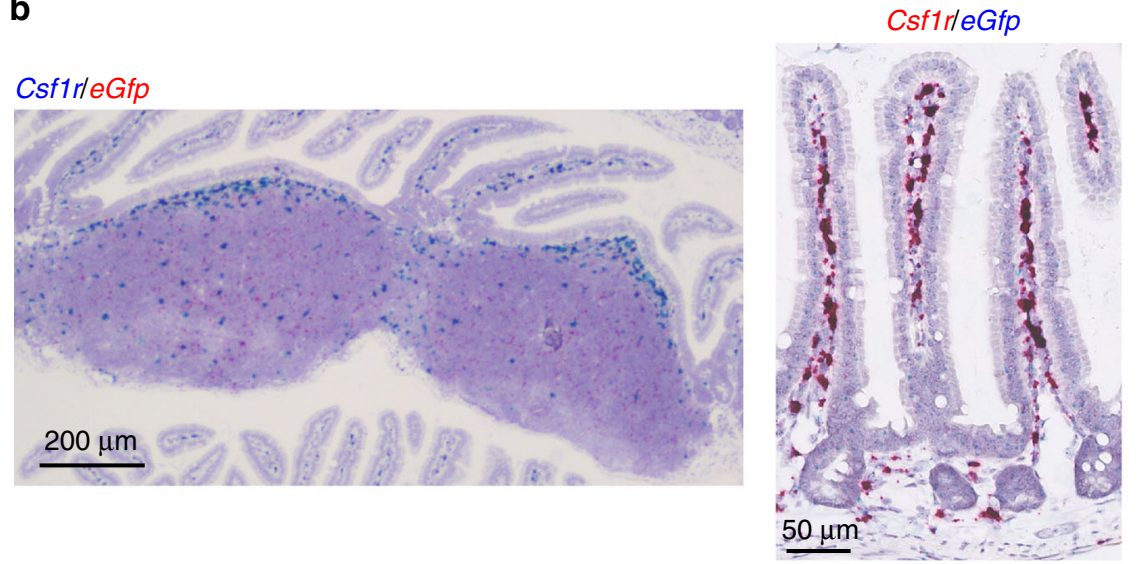

Csf1rlEmr1

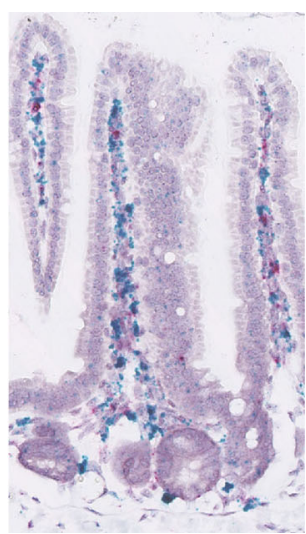

C

Control
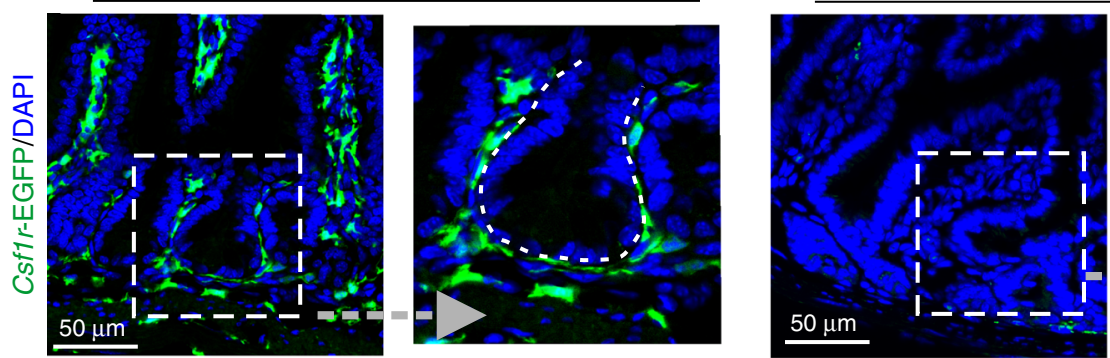

Anti-CSF1R

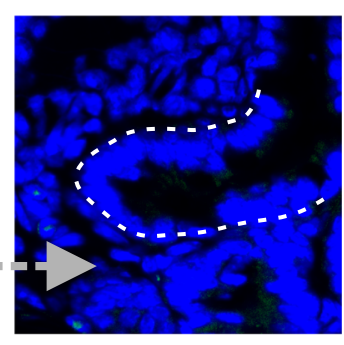

Fig. 4 Macrophages are the only cells which express CSF1R within intestinal crypts. a IHC analysis shows Csf1r-EGFP+CD68 ${ }^{+}$macrophages are in direct intimate contact with the crypt epithelial cell layer. Scale bar, $20 \mu \mathrm{m}$. b Left-hand panel shows the distribution of Csflr (blue) and eGfp (red) mRNA expression in Peyer's patches. Scale bar, $200 \mu \mathrm{m}$. In the gut wall Csfir (red, middle panel; blue, right-hand panel) and eGfp (blue) mRNA expression was only expressed by macrophages (Emr1, red; right-hand panel). Scale bar, $50 \mu \mathrm{m}$. c IHC analysis shows that in the crypts and gut wall of Csf1r-EGFP mice, Csf1r-EGFP ${ }^{+}$macrophages (green) were depleted after CSF1R blockade. Sections in a, c were counterstained with DAPI to detect cell nuclei (blue). Broken lines indicate the crypt epithelial cell layer boundary. Scale bar, $50 \mu \mathrm{m}$. Representative images are shown from 3 to 4 intestinal segments from 4 to 8 mice/ group, from three independent experiments

individual cell populations derived from them and in deep CAGE sequence data from the FANTOM consortium (Supplementary Fig. 3-5 $)^{25-27}$. To confirm the lack of expression, we performed RNA in situ hybridization analysis on small intestines from naïve C57BL/6J wild-type mice. Csf1r mRNA expression was not detected in epithelial cells within the crypts or anywhere within the gut epithelium (Fig. 3f). To further confirm that Csf1r is not expressed functionally in epithelial cells at any stage, we applied a conditional knockout using Villin-Cre, to target Csf1r-deficiency to epithelial cells, including Paneth cells: Csf1 $\mathrm{r}^{\Delta \mathrm{IEC}}$ mice. The same promoter was used previously to drive tamoxifen-inducible Cre-mediated deletion of Csf1 $r^{9}$. Vil1-Cre mice and Csf1 $\mathrm{r}^{\mathrm{FL} / \mathrm{FL}}$ mice were used as transgenic controls. The numbers of lysozymeexpressing Paneth cells were unchanged in the intestinal crypts of Csfl $r^{\Delta \mathrm{IEC}}$ mice compared to Vill-Cre and Csf1 $\mathrm{r}^{\mathrm{FL} / \mathrm{FL}}$ transgenic control mice (Fig. 3g, h). Paneth cell morphology and expression of Defal were also unaffected in the intestines of Csf1 $\mathrm{r}^{\Delta \mathrm{IEC}}$ mice
(Supplementary Fig. 6). These data do not support the proposed direct role for CSF1R signaling in small intestinal crypt homeostasis ${ }^{9,10}$. Instead, an indirect role is supported by the close association between the CSF1R-expressing macrophages and the crypt epithelium.

In the vicinity of the intestinal crypt, Csf1r-EGFP expression was detectable in $\mathrm{CD}^{+} 8^{+}$macrophages in direct intimate contact with the epithelial cell layer with frequent extended processes contacting the epithelial cells within it (Figs. $3 \mathrm{~d}$ and 4a; Supplementary Fig. 7; Supplementary movie 1). Two-color RNA in situ hybridization analyses confirmed that the EGFP expression observed in the intestine and Peyer's patches directly mirrored the cellular distribution of Csf1r mRNA expression, and that the Csf1r-expressing cells also expressed the macrophage marker Emr1 (Fig. 4b). Furthermore, in the crypts and gut wall of Csf1r-EGFP mice, Csf1r-EGFP ${ }^{+}$ macrophages were depleted after CSF1R blockade (Fig. 4c). 
Consistent with the proposed indirect role of macrophages, as shown in Fig. 2d, e, the restoration of lysozyme expression in Paneth cells coincided with the reappearance of macrophages in the gut following the removal of the anti-CSF1R mAb treatment.
Macrophage depletion indirectly impairs crypt homeostasis. As noted earlier, Paneth cells help maintain the Lgr5 ${ }^{+}$intestinal stem cells in the crypt niche through the provision of homeostatic factors ${ }^{2}$. Following prolonged CSF1R blockade a reduction in Lgr5 mRNA expression by the intestinal stem cells at the a

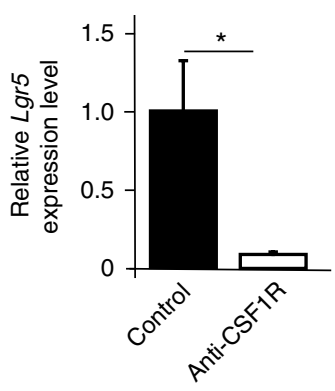

C

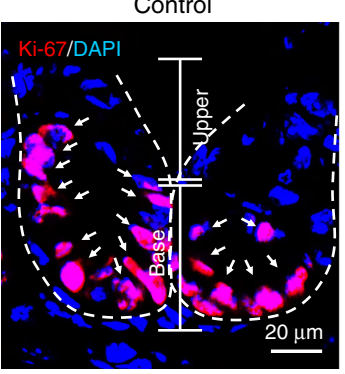

e
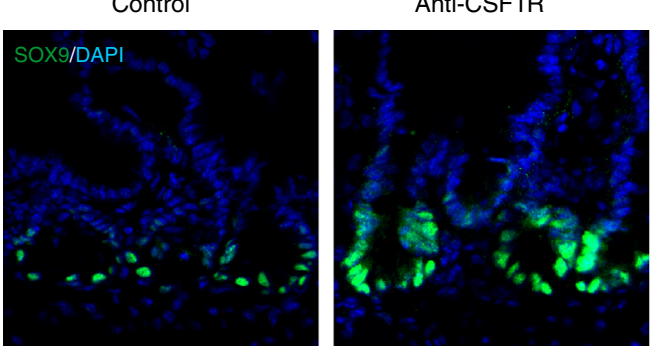

$\mathbf{g}$

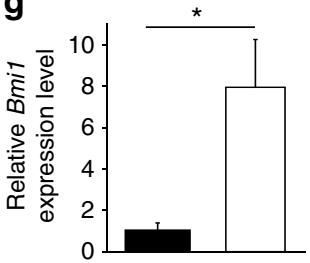

h

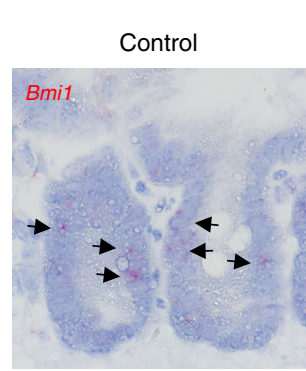

b

\section{Control}

Anti-CSF1R
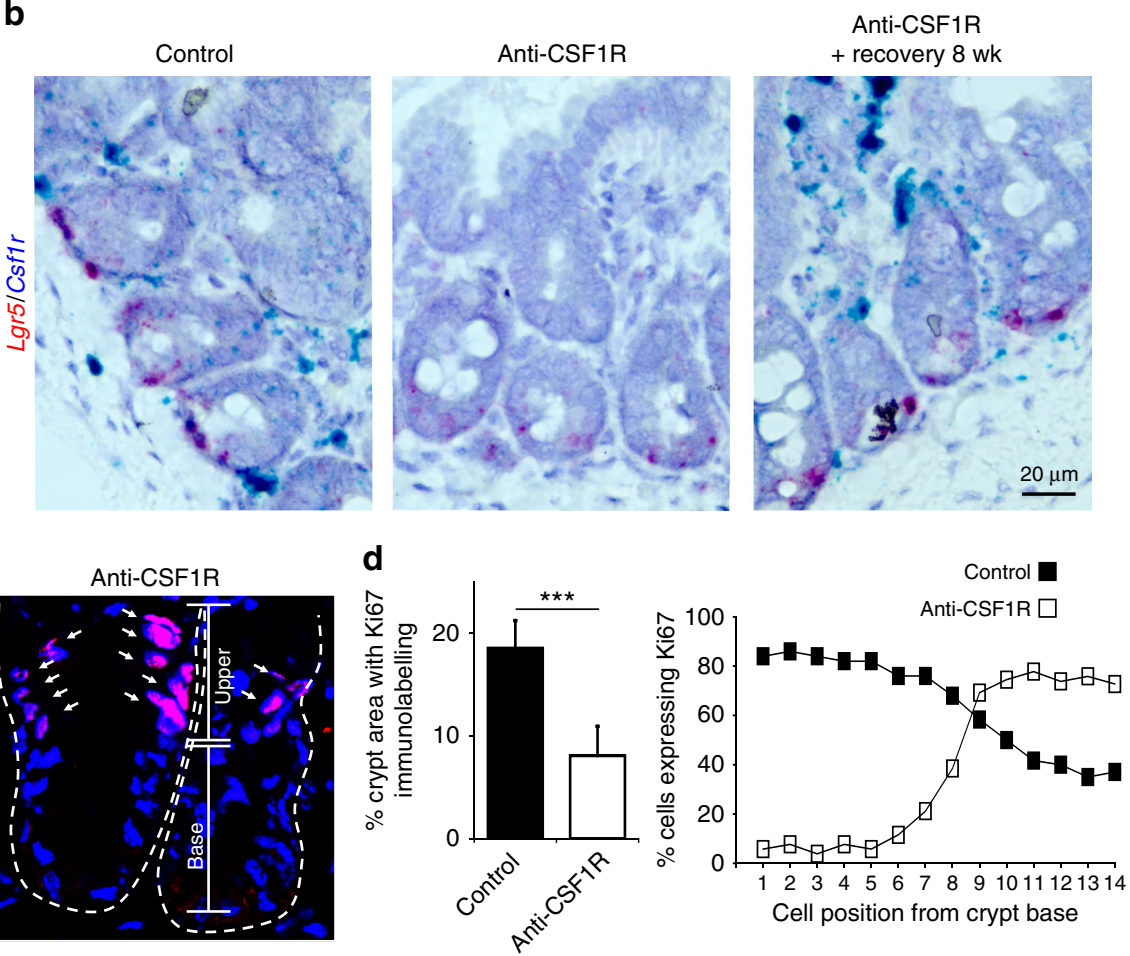

f

+ recovery 8 wk
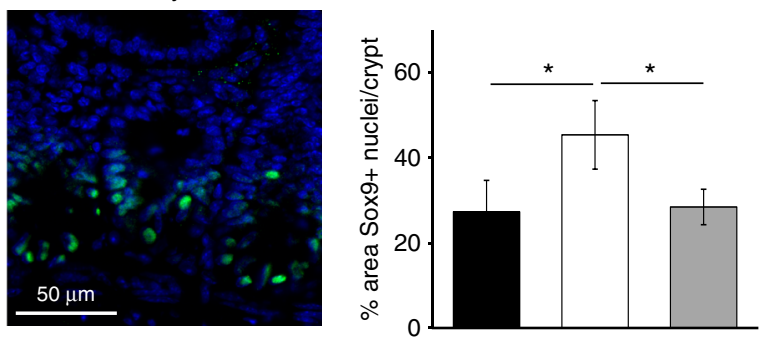

Control

$\square$ Anti-CSF1R

$\square$ Anti-CSF1R + recovery 8 wk

$\square$ Anti-CSF1R

Anti-CSF1R + recovery $8 w k$
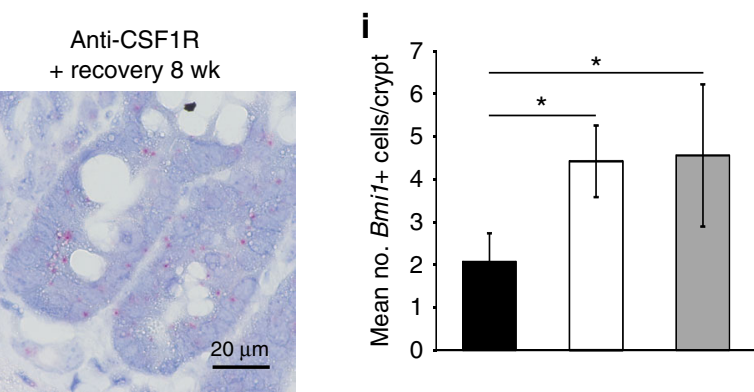
base of the intestinal crypts was observed (Fig. 5a, b). The effects of prolonged CSF1R blockade on Lgr5 expression were also transient and returned to similar levels as observed in control mice following the $8 \mathrm{wk}$ recovery period after antiCSF1R mAb treatment (Fig. 5b). The reduced Lgr5 expression coincided with a significant reduction in $\mathrm{Ki}-67^{+}$proliferating cells in the base of the intestinal crypt (Fig. $5 c$, d). Independent studies have shown that a reserve stem cell population within the intestinal crypts can compensate for the complete loss of Lgr5-expressing cells ${ }^{28,29}$. In the steady state these cells are mostly restricted to the " +4 " cell position within the crypt adjacent to the upper most Paneth cell ${ }^{30}$. These reserve intestinal stem cells express SOX9 and BMI1 and expand during crypt injury to help maintain gut epithelial homeostasis 28,29 . Consistent with this activity, following the reduction in Lgr5 expression after prolonged CSF1R blockade, cell proliferation $\left(\mathrm{Ki}-67^{+}\right.$cells) was displaced to the upper crypt region in tissues from anti-CSF1R-treated mice (Fig. 5c, d). A significant increase in the abundance of SOX9 ${ }^{+}$ cell nuclei (Fig. 5e, f) and Bmil mRNA expression (Fig. 5g-i) was also observed in the crypts after prolonged CSF1R blockade, suggesting expansion of the reserve intestinal stem cell pool.

The expansion of the reserve intestinal stem cells following CSF1R blockade is consistent with our previous demonstration that villous length was significantly longer in the small intestines of mice treated with anti-CSF1R mAb ${ }^{13}$. The large intestine lacks Paneth cells, but in the colon regenerating islet-derived family member 4 (REG4)-expressing deep secretory cells appear to maintain crypt homeostasis ${ }^{31}$. In the current study, colonic crypt length was also significantly extended in the large intestines of anti-CSF1R mAb mice, implying a similar role for macrophages in the maintenance of the colonic crypt niche (Supplementary Fig. 8).

To further eliminate direct roles of CSF1R signaling in the gut epithelium, in vitro enteroids (comprising only of epithelial cell lineages) were prepared from small intestinal crypts of C57BL/6J wild-type mice and treated with either anti-CSF1R mAb, or recombinant CSF1-Fc. Low levels of expression of macrophageassociated genes and Csflr were detected in enteroids freshly prepared from the intestine (passage 0; Fig. 6a), but expression of these genes was undetectable after subsequent passages (passages 1-3; Fig. 6a). Neither anti-CSF1R treatment, nor addition of exogenous CSF1, had any significant effect on the growth of these enteroids throughout multiple serial passages (Fig. 6b, c), nor the expression of lysozyme by Paneth cells, and Lgr5 and Olfm4 (encoding olfactomedin-4) by the intestinal stem cells within them (Fig. 6d). These data also suggest that the growth factors added to these cultures to sustain the enteroids in vitro can substitute for the in vivo requirement for macrophages. Consistent with the absence of Csfir mRNA expression by any gut epithelial cell lineage, the specific ablation of Csf1r only in the gut epithelium similarly did not affect the growth of enteroids prepared from Csf1r $\triangle \mathrm{IEC}$ mice, when compared to those prepared from Vil1-Cre or Csf1 $\mathrm{r}^{\mathrm{FL} / \mathrm{FL}}$ mice transgenic control mice (Fig. 6e, f).

Enteroids prepared from crypts of mice depleted of Lgr5expressing intestinal stem cells grow with a similar efficiency as those prepared from control mice due to the increased activity of the reserve intestinal stem cell population ${ }^{28}$. In the current study, the growth of enteroids prepared from the crypts from mice treated with anti-CSF1R mAb was similar to those derived from control-treated mice (Fig. $6 \mathrm{~g}, \mathrm{~h}$ ). This suggests that the reserve intestinal stem cell population is similarly able to compensate for the reduction in Lgr5-expressing stem cells in the crypt following prolonged CSF1R blockade.

The functions of the crypt-associated macrophages could be mediated indirectly, through impacts observed on Paneth cells, or directly by acting upon the Lgr $5^{+}$stem cells. Several recent papers implicate macrophages as the source of Wnt family members that control epithelial differentiation following injury ${ }^{32,33}$. We therefore compared the expression of mRNA encoding important genes involved in Wnt-signaling and the homeostatic maintenance of the intestinal crypts in microarray datasets derived from distinct mononuclear phagocyte populations isolated from the small intestine. This analysis revealed that small intestinal macrophages specifically expressed Wnt4 and Rspol (R-spondin, the ligand for Lgr5), and IHC analysis confirmed the expression of Wnt 4 by $\mathrm{CD}^{+} 8^{+}$macrophages in the gut wall (Supplementary Fig. 9).

\section{Macrophage depletion indirectly impairs M-cell differentia-} tion. The indirect effect of prolonged CSF1R blockade on intestinal crypt homeostasis disturbs the subsequent differentiation of several epithelial cell lineages. For example, the density of goblet cells in the gut epithelium was significantly increased in Csf $1 r^{-1-}$ mice or Csf1 $1^{\text {op/op }}$ mice ${ }^{10}$ or following prolonged CSF1R blockade $^{13}$. M cells are a unique subset of epithelial cells which are specialized for the transcytosis of microorganisms and particulate antigens across the gut epithelium into the Peyer's patches $^{18}$. M cells do not express Csf1r or translate CSF1R protein (Fig. 7a, b, Supplementary Fig. 10), but since they also derive from Lgr5-expressing intestinal stem cells ${ }^{17}$ we reasoned that

\footnotetext{
Fig. 5 Prolonged anti-CSF1R treatment impairs crypt homeostasis. Mice were treated with anti-CSF1R mAb or control-lgG (control) for 6 weeks before analysis. A parallel group of mice were allowed to recover for $8 \mathrm{wk}$ following anti-CSF1R mAb treatment. a Comparison of Lgr 5 mRNA expression in isolated intestinal crypts from anti-CSF1R (open bars) or control-treated (closed bars) mice by RT-qPCR analysis. Bars represent mean \pm SEM derived from four mice/group. ${ }^{\star} P<0.05$, two-tailed unpaired Student's $t$-test. $\mathbf{b}$ Two-color mRNA in situ hybridization analysis confirmed a reduction in Lgr5 mRNA expression (red) at the base of intestinal crypts coincident with the ablation of Csf1r mRNA (blue) expressing macrophages. Representative images from three mice/group are shown. Scale bar, $25 \mu \mathrm{m}$. c Detection of proliferating Ki-67+ cells (red) in intestinal crypts by IHC. Sections counterstained with DAPI to detect nuclei (blue). Broken lines indicate the crypt epithelial cell layer boundary. Scale bar, $20 \mu \mathrm{m}$. d Morphometric analysis of (left) the magnitude of Ki-67-specific immunostaining in intestinal crypts, and (right) the distribution of Ki-67-expression within the crypt. Data represent mean \pm SD derived from 5 to 9 sections from four mice/group. ${ }^{\star \star} P<0.001$, two-tailed unpaired Student's $t$-test. e IHC detection of SOX9 expression (green) in intestinal crypts. Sections were counterstained with DAPI to detect cell nuclei (blue). Scale bar, $50 \mu \mathrm{m}$. f Morphometric analysis confirmed that following prolonged CSF1R blockade the $\%$ area of the crypts with SOX 9 + cell nuclei was significantly increased. Bars represent mean \pm SD derived from four mice/group with 50-100 crypts/mouse, and are representative of data from two independent experiments. ${ }^{\star} P<0.05$, one-way ANOVA with Tukey's post hoc test. $\mathbf{g}$ RT-qPCR analysis of Bmil mRNA expression in isolated intestinal crypts from anti-CSF1R or control-treated mice. Bars represent mean \pm SEM derived from four mice/group. ${ }^{\star} P<0.05$, two-tailed unpaired Student's $t$-test. $\mathbf{h}$ mRNA in situ hybridization analysis revealed an increase in the abundance of $B m i 1-$ expressing cells (red, arrows) in the intestinal crypts of anti-CSF1R-treated mice. Scale bar, $20 \mu \mathrm{m}$. i Mean no. Bmil-expressing cells/crypt. Bars represent mean \pm SD derived from four mice/group with 40-50 crypts/mouse. ${ }^{\star} P<0.05$, one-way ANOVA with Tukey's post hoc test
} 
prolonged CSF1R blockade could indirectly affect M-cell differentiation. Mature, glycoprotein 2 (GP2)-expressing M cells with characteristic basolateral pockets were abundant in the FAE of Peyer's patches from control-treated mice, but their density was reduced after prolonged CSF1R blockade (Fig. $7 \mathrm{c}, \mathrm{d} ; P<0.001$ ). The size of the FAE was not affected by prolonged CSF1R blockade (Fig. 7e). Like the other effects of anti-CSF1R-mAb treatment, $\mathrm{M}$ cell differentiation was restored within $8 \mathrm{wk}$ following cessation of treatment (Supplementary Fig. 11A, B), coincident with the restoration of the macrophages and crypt homeostasis. M cell density was unaffected in the FAE of Peyer's patches from Csf1 $\mathrm{r}^{\triangle \mathrm{IEC}}$ mice (Supplementary Fig. 11C, D) excluding a direct role for CSF1R signaling in the gut epithelium in $\mathrm{M}$ cell differentiation. Consistent with our previous study ${ }^{13}$, a

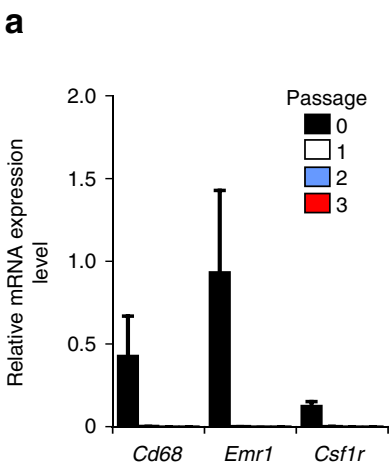

b

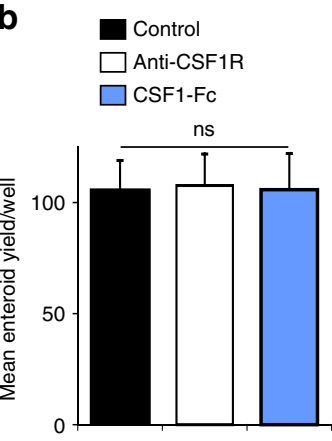

C
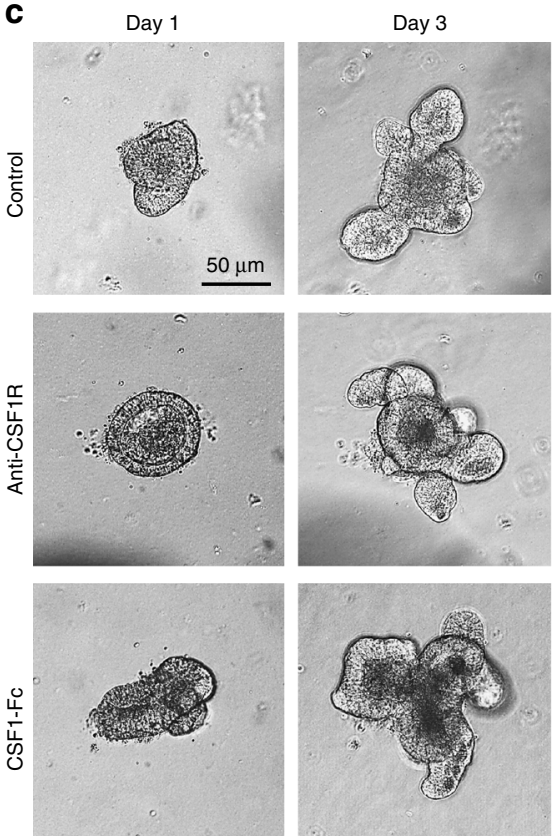

e

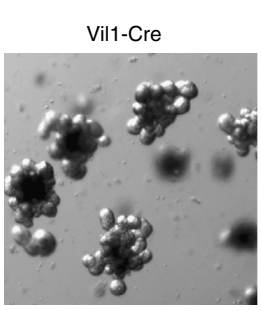

g

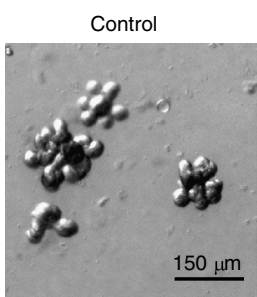

Csf1r FL/FL

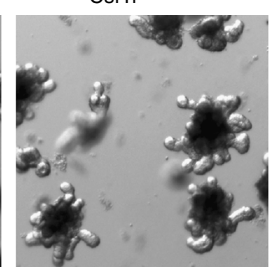

Anti-CSF1R

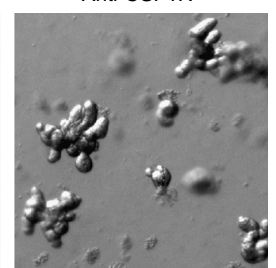

Day 5
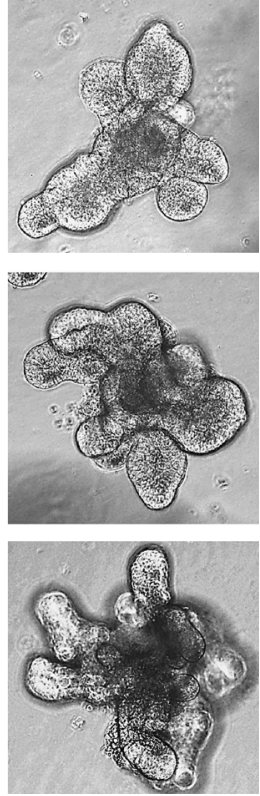

Csf1 $1 \mathrm{r}^{\Delta \mathrm{IEC}}$

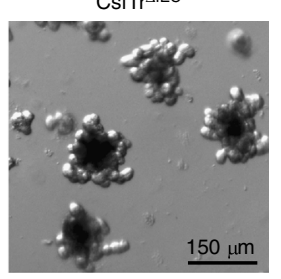

h

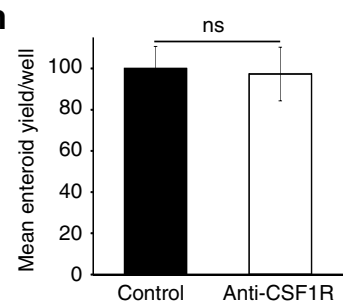

d
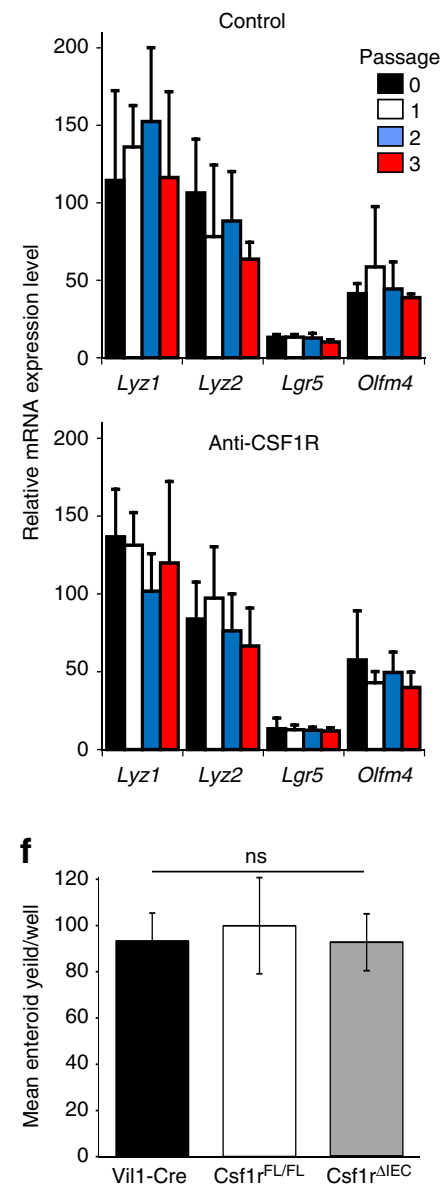
significant increase in the density of goblet cells was observed in the FAE of Peyer's patches in the small intestine after prolonged CSF1R blockade (Fig. 7c, d; recognized by their morphology, binding of UEA-1 and absence of GP2; $P<0.001$ ). A significant increase in goblet cell density was also observed in the colons of the same mice (Supplementary Fig. 8).

We next determined whether the reduced density of $\mathrm{GP}^{+} \mathrm{M}$ cells in anti-CSF1R-treated mice correlated with a reduced functional ability to acquire particulate antigens from the gut lumen. The assessment of the uptake of fluorescent latex beads injected into ligated intestinal loops is a useful quantitative method to compare the functional activity of $M$ cells in vivo ${ }^{20,34,35}$. In the Peyer's patches of control-treated mice numerous beads had been transcytosed across the FAE into the subepithelial dome (SED) region (Fig. 7f). However, following prolonged CSF1R blockade this ability was reduced demonstrating that the functional capacity of $\mathrm{M}$ cells to acquire particulate antigens from the gut lumen was adversely affected (Fig. 7f, g; $P<$ 0.001 ).

The differentiation of $\operatorname{Lgr} 5^{+}$stem cell-derived daughter cells into functionally mature $\mathrm{M}$ cells requires additional stimulation from the mesenchymal stromal cells within the SED region beneath the FAE. In the GALT the production of the cytokine, receptor activator of $N F-\kappa B$ ligand (RANKL), by these subepithelial mesenchymal stromal cells is the critical factor which stimulates the differentiation of RANK-expressing (the receptor for RANKL) enterocytes into $M$ cells ${ }^{35}$. This differentiation process can be divided into distinct stages based on the expression particular $\mathrm{M}$-cell markers ${ }^{18,20}$. Immature, differentiating $\mathrm{M}$ cells express annexin A5 (Anxa5) and Marcksl1. The intrinsic expression of the ETS transcription factor SpiB by these differentiating $M$ cells is critical for their subsequent differentiation into functionally mature $M$ cells, which in addition to GP2, also express CCL9 and SGNE1 ${ }^{18,20}$. Prolonged CSF1R blockade affected M-cell development prior to their differentiation into the immature stage as the expression of all of these immature and mature $\mathrm{M}$ cell markers was reduced by the treatment (Fig. 8).

Although CSF1 can regulate RANKL expression by osteoclasts $^{36}$, the effects of prolonged CSF1R blockade on M-cell status observed here were not due to impaired RANKL-RANK stimulation. High levels of RANKL were detected on the subepithelial mesenchymal stromal cells in the Peyer's patches of anti-CSF1R-treated and control-treated mice (Fig. 9a). Consistent with this observation, independent mRNA sequencing data from subepithelial mesenchymal stromal (podplanin ${ }^{+}$MAdCAM-1 ${ }^{-}$) cells and mesenchymal marginal reticular (podplanin ${ }^{+}$MAdCAM- $1^{+}$) cells enriched from Peyer's patches ${ }^{37}$ indicates that neither cell population expresses Csf1r (Fig. 9b). The expression of Tnfsf11 (encoding RANKL), Tnfrsf11a (encoding RANK), and Tnfrsfilb (encoding the RANKL decoy receptor osteoprotegerin, OPG) in Peyer's patches was also unaffected by prolonged CSF1R blockade (Fig. 9c). The CCL20mediatiated attraction of CD11c-expressing B cells towards the FAE has also been shown to aid the maturation of $M$ cells from immature Anxa5 ${ }^{+}$precursors ${ }^{34,38}$. However, CCL20 expression in the FAE (Fig. 9d) and the attraction of " $M$ cell-inducing"

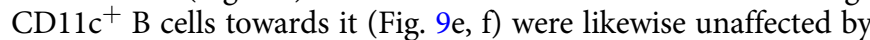
prolonged CSF1R blockade. These data are consistent with the demonstration that $\mathrm{M}$ cell development was adversely affected prior to their differentiation into the immature Anxa5 ${ }^{+}$stage (Fig. 8).

Taken together, these data demonstrate that the effects of prolonged CSF1R blockade on M-cell development were due to disturbances in crypt homeostasis. These data reveal a previously unrecognized, indirect requirement for macrophages in the maintenance of $M$ cells in Peyer's patches through their essential role in the constitutive maintenance of Paneth cells and Lgr5 stem cells in the FAE-associated crypts.

\section{Discussion}

Here we show that crypt-associated macrophages are constitutively required to maintain the crypts in the small intestine. Their depletion following prolonged CSF1R blockade affects the differentiation of Paneth cells and $\mathrm{Lgr}^{+}$stem cells in the crypt, notably resulting in a change in the balance between goblet cell and $M$ cell differentiation. These data suggest that modification of the phenotype or abundance of macrophages in the gut wall, for example after pathogen infection, could adversely affect the development of the intestinal epithelium and the ability to sample particulate antigen from the gut lumen.

In the small intestine the $\operatorname{Lgr} 5^{+}$stem cells within the base of the crypts are interspersed between terminally differentiated Paneth cells. The Paneth cells provide essential stimuli for the constitutive maintenance of the $\operatorname{Lgr}^{+}$stem cells including Wnt 3 A, EGF, TGFa and the Notch ligand Dll4 ${ }^{2,39,40}$, and the loss of Paneth cells results in a significant reduction in $\operatorname{Lgr}^{+}$stem cells ${ }^{2}$. In the current study, at least part of the effect of macrophage depletion probably occurs through the impact on Paneth cells, which is likely responsible for the reduction of $\mathrm{Lgr}^{+}$stem cells. However, it is possible that the macrophages also interact directly with the stem cells, as the proliferation of $\mathrm{Lgr}^{+}$stem cells is not entirely Paneth cell dependent. In Paneth cell deficient Atoh $1^{-1-}$ mice some Lgr5 $^{+}$stem cells can survive and proliferate ${ }^{41}$. Furthermore, the specific deletion of Wnt signals only in the gut epithelium or in subepithelial myofibroblasts does not affect the status of Paneth cells or Lgr5 ${ }^{+}$stem cells, indicating the presence of an alternative cellular source of support ${ }^{42,43}$. Wntsignaling in the intestinal stem cell niche requires direct cell-tocell contact $^{39}$. Given the tight association of macrophages with the intestinal crypt, we hypothesize that in the specific absence of

\footnotetext{
Fig. 6 Anti-CSF1R-treatment or CSF1-stimulation do not directly affect crypt homeostasis. a RT-qPCR analysis shows enteroids prepared from the small intestines of untreated C57BL/6J mice express negligible levels of Cd68, Emr1, and Csf1r. Expression of these genes is undetectable after passage. Data represent mean \pm SEM from triplicate cultures. b, c Enteroids were prepared from the small intestines of $\mathrm{C} 57 \mathrm{BL} / 6 \mathrm{~J}$ mice and at passage 3 were cultivated in the presence or absence of anti-CSF1R mAb $(10 \mu \mathrm{g} / \mathrm{ml})$, control rat lgG $(10 \mu \mathrm{g} / \mathrm{ml})$ or CSF1-Fc $(500 \mathrm{ng} / \mathrm{ml})$. b Mean enteroid yield and size at day 5 after treatment. Data represent mean \pm SD from eight wells/treatment. Enteroid yield; NS Kruskal-Wallis one-way ANOVA. Enteroid size; NS one-way ANOVA with Tukey's post hoc test. c Representative morphology of the enteroids at intervals after treatment. Scale bar, $50 \mu \mathrm{m}$. d RT-qPCR analysis shows the expression of Lyz1, Lyz2, Lgr5, and Olfm4 in enteroids was unaffected by treatment with anti-CSF1R mAb. Data represent mean \pm SEM from triplicate cultures. e Representative images of day 7 enteroids prepared from the crypts of Csf1r ${ }^{\Delta \mathrm{IEC}}$, Vil1-Cre and Csf1r $\mathrm{FL}^{\mathrm{FL}} \mathrm{mice}$. Scale bar, $150 \mu \mathrm{m}$. $\mathbf{f}$ The mean yield of enteroids prepared from the crypts of $\mathrm{Csf1} \mathrm{r}^{\Delta \mathrm{EEC}}$, Vil1-Cre and $\mathrm{Csf1} \mathrm{r}^{\mathrm{FL}} / \mathrm{FL}$ mice on day 7 of cultivation was similar. Data represent mean $\pm \mathrm{SD}$ from enteroids prepared from 3 mice/group, 9 wells/mouse. ns, one-way ANOVA with Tukey's post hoc test. $\mathbf{g}$ Representative images of day 5 enteroids prepared from the crypts of mice treated with anti-CSF1R mAb or control-IgG (control) for 6 weeks. Scale bar, $150 \mu \mathrm{m}$. $\mathbf{h}$ The mean yield of enteroids prepared from the crypts of mice from each group was similar. Data represent mean \pm SEM from 14 to 16 wells of organoids from four mice/group. NS twotailed unpaired Student's t-test
} 
Paneth cells, factors derived from the crypt-associated macrophages are able to provide homeostatic support to the $\mathrm{Lgr}^{+}{ }^{+}$stem cells.

In contrast to conclusions from previous reports, ${ }^{9,10}$, we show that prolonged CSF1R blockade did not lead to the loss of Paneth cells, as cells with abundant secretory cytoplasmic granules were evident in the crypts of treated mice. Instead, we show that cryptassociated macrophages provide factors which help to maintain
Paneth cell differentiation. Paneth cells release antimicrobial products such as lysozyme and $\alpha$-defensins alongside homeostatic factors such as Wnt3 which are essential for the maintenance of the Lgr5 $^{+}$intestinal stem cells ${ }^{2}$. In the absence of the cryptassociated macrophages the expression of lysozyme and Wnt 3 by Paneth cells was significantly reduced. The release of antimicrobial products by Paneth cells helps to prevent bacterial translocation across the gut epithelium and protects the crypts
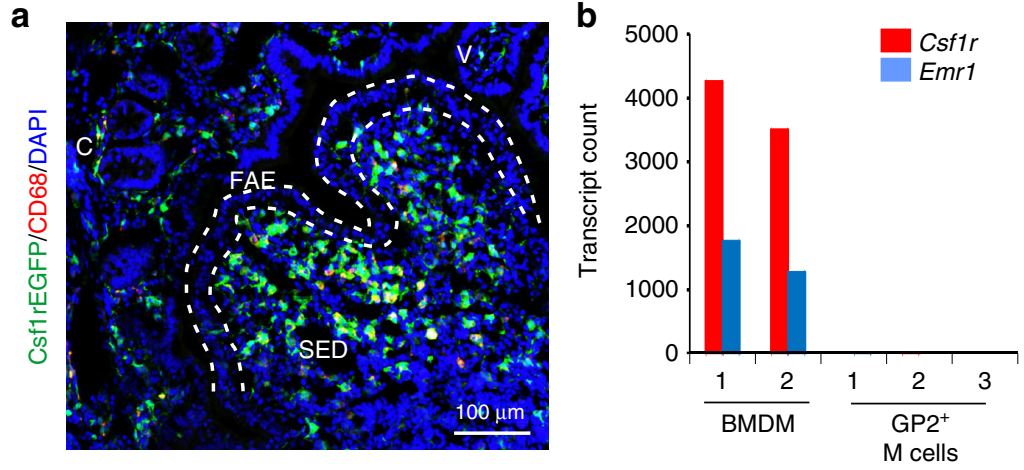

C

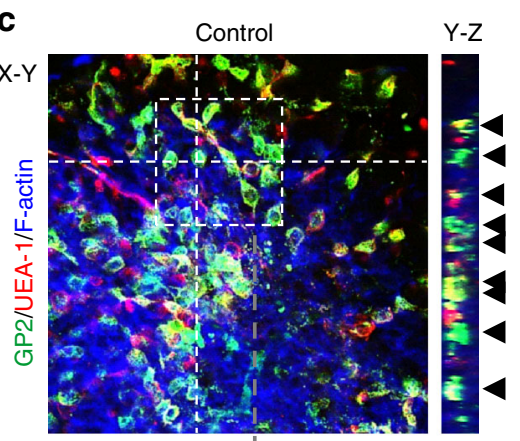

$\mathrm{X}-\mathrm{Z}$

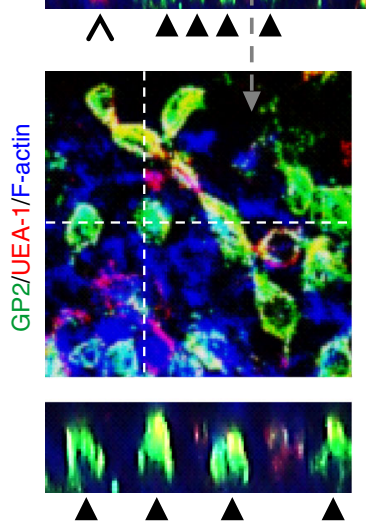

f

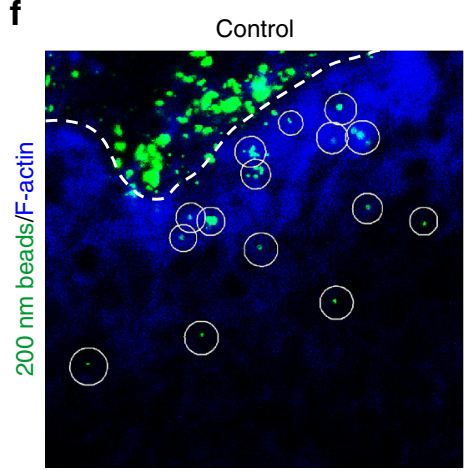

Y-Z

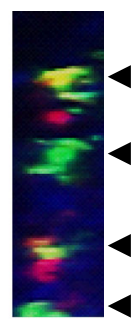

(

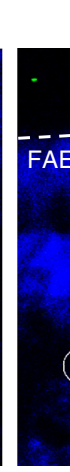

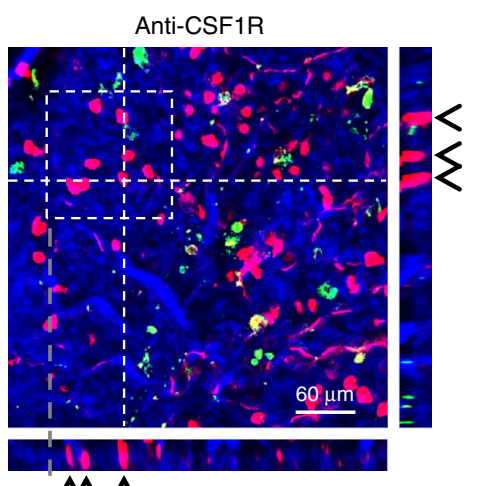
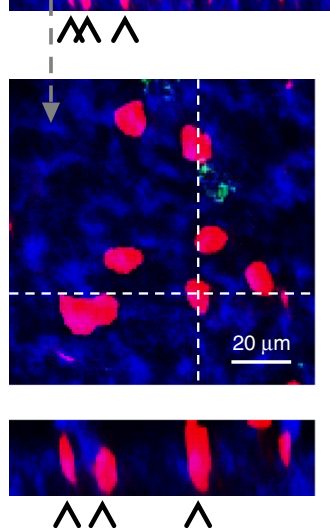

$\wedge \wedge \wedge$

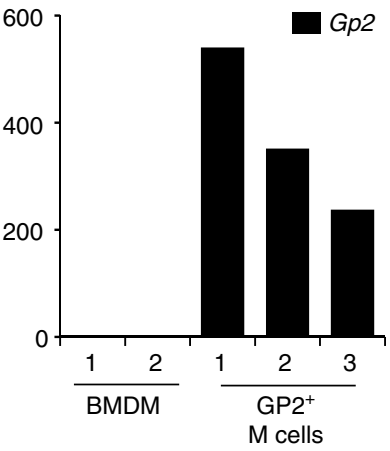

d

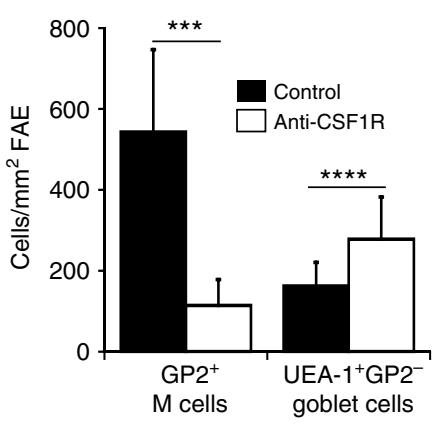

e

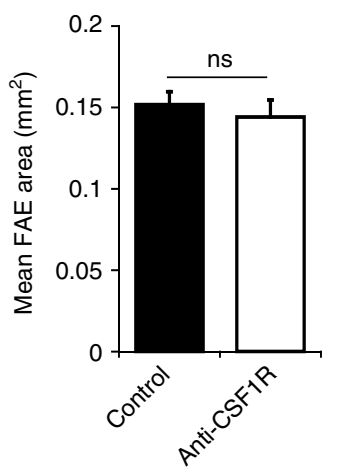


from bacterial infection. Whereas Paneth cell-derived $\alpha$-defensins have selective antimicrobial activity against pathogenic bacteria, they have limited or no activity against commensal bacteria ${ }^{44}$. Defects in Paneth cells or their production of antimicrobial factors can result in intestinal microbiota dysbiosis, expansion of pathogenic noncommensals and susceptibility to enteric infections ${ }^{44,45}$. The expression of Defa1 by Paneth cells was unaffected by prolonged CSF1R blockade, implying that the protection of the crypts against pathogenic bacteria was maintained. However, the reduced Paneth cell-derived lysozyme expression may cause microbiota dysbiosis and the expansion of certain commensal populations such as Escherichia coli ${ }^{46}$.

The factors produced by the crypt-associated macrophages which provide this essential support to Paneth cells are not known. The clear apposition of macrophages with the intestinal crypt stem cells strongly suggests a trophic interaction, perhaps akin to their relationship with haemopoietic stem cells in the bone marrow ${ }^{47,48}$, or with osteoblasts on the surface of bone ${ }^{49}$. Wnt signals can separately stimulate both $\mathrm{Lgr}^{+}$stem cells and Paneth cells ${ }^{40}$, and Wnt-signal expression by macrophages is widely documented ${ }^{32,50}$. Bone morphogenetic protein signaling is also essential for the maintenance of the $\mathrm{Lgr}^{+}{ }^{+}$stem cell population $^{51}$, and there may be parallels with the trophic interactions between macrophages and neurons in the muscularis externa ${ }^{15,16}$. Indeed, the same reciprocal relationship between macrophages and CSF1-producing cells may occur, since Paneth cells have been reported to be sites of CSF1-synthesis ${ }^{52}$. Paneth cells may also provide EGF to the $\mathrm{Lgr}^{+}{ }^{+}$stem cells ${ }^{2}$, and here also, CSF1dependent macrophages could provide an additional source of growth factor ${ }^{53}$. In overview, it is unlikely that the trophic function of crypt-associated macrophages relies on a single factor. In support of this we found that the expression of two candidate factors, Wnt4 and Rspol was enriched in small intestinal macrophages implying a potential role. Since R-spondin 1 is a Wnt agonist, the ligand for Lgr $5^{54}$ and is an essential factor required for the in vitro cultivation of enteroids ${ }^{1}$ it is plausible that the macrophages interact directly with, and provide homeostatic support to, both Paneth cells and intestinal stem cells.

Our data contradict the view that Paneth cells express functional CSF1R ${ }^{9,10}$. One group has suggested that CSF1R can also be expressed by renal epithelial cells during regeneration in response to injury ${ }^{55}$, but others have demonstrated that in this system the impacts of CSF1 treatment are also mediated through macrophages $^{56}$. The growth of enteroids from Csfir $1 r^{-1-}$ mice has been reported to be impaired ${ }^{9}$. However, in the current study enteroids prepared from Csfir $\mathrm{r}^{\mathrm{\Delta IEC}}$ mice grew equally well, and neither CSF1, nor anti-CSF1R mAb treatment, has any effect in vitro. The reasons for the discrepancies between these studies is uncertain. The published studies relied on the use of a polyclonal anti-CSF1R antibody to detect the protein ${ }^{10}$ and a tamoxifen- inducible Cre to initiate conditional deletion of $C s f 1 r^{9}$. The former might not be specific, while in the latter case, direct effects of tamoxifen on macrophages may not be excluded. Although crypt homeostasis was disturbed following prolonged CSF1R blockade, the growth of enteroids prepared from the intestines of these mice in the current study was equivalent to those from controls. Independent studies have shown that enteroids prepared from the crypts of mice which were depleted of $\mathrm{LgrS}^{+}$intestinal stem cells are similarly able to grow and proliferate due to the expansion of the reserve intestinal stem cell population ${ }^{28}$, which is insensitive to Wnt pertubations ${ }^{29}$. We observed a similar expansion of the $\mathrm{SOX}{ }^{+} \mathrm{BMI}^{+}$reserve intestinal stem cell population following prolonged CSF1R blockade, suggesting that these cells were similarly attempting to restore crypt homeostasis in the absence of macrophages.

Disturbances to the homeostasis of the crypt can significantly affect the proportions of epithelial cell lineages in the gut epithelium. The $\mathrm{Lgr}^{+}$stem cells produce highly proliferative transit-amplifying daughter cells which differentiate into one of several specialized intestinal epithelial cell lineages depending on the additional stimulation they receive $e^{20,35,57}$. These include secretory cell lineages such as enteroendocrine cells, goblet cells, Paneth cells or tuft cells. For example, the indirect effects of prolonged CSF1R blockade on the crypt enhances goblet cell differentiation in the small intestine $\mathrm{e}^{10,13}$ and colon. The antigen-sampling $\mathrm{M}$ cells also derive from the $\mathrm{Lgr}^{+}$stem cells in the crypts surrounding the FAE which differentiate into mature, functional $\mathrm{GP}^{+}{ }^{+} \mathrm{M}$ cells upon RANKL-stimulation from subepithelial mesenchymal stromal cells $s^{17,20,35,37}$. The density of $\mathrm{M}$ cells in Peyer's patches was reduced after prolonged CSF1R blockade, as was their ability to acquire particulate antigens from the gut lumen. The effects of anti-CSF1Rtreatment on the development of $\mathrm{M}$ cells were indirect since these cells also do not express CSF1R. Instead we reveal a previously unrecognized, indirect requirement for macrophages in the maintenance of $\mathrm{M}$ cells in Peyer's patches through their essential role in the constitutive maintenance of the FAEassociated crypts.

The link between lamina propria macrophages and $\mathrm{M}$ cells provides a potential feedback loop to ensure some coordination of antigen sampling, innate immune defence and antigen presentation. The pharmacological blockade of CSF1R signaling has also been proposed as a means to modulate certain cancers, and inflammatory, autoimmune and bone disease ${ }^{58}$. Prolonged CSF1R blockade could indirectly compromise intestinal crypt homeostasis and the ability to sample particulate antigens/ pathogens from the gut lumen. Paneth cell-dysfunction is evident in patients who have Crohn's disease (CD) with small intestinal involvement and may be a consequence of inadequate Wnt-ligand stimulation by monocytes ${ }^{5}$. Monocytes from

Fig. $7 \mathrm{M}$ cells are dramatically reduced in Peyer's patches after prolonged CSF1R blockade. a Histological analysis of Csf1r-EGFP mice shows CSF1R (green) is not expressed by epithelial cells in Peyer's patches. FAE, follicle-associated epithelium; SED, subepithelial dome; dotted lines indicate the FAE boundary. Scale bar, $100 \mu \mathrm{m}$. b Expression of Csf1r (middle panel) and Gp2 in individual deep CAGE sequence datasets of mouse bone marrow-derived macrophages (BMDM) and $M$ cells $^{25}$. Csf1r mRNA is undetectable in M cells. c-g Mice were treated with anti-CSF1R mAb or control-lgG (control) for 6 weeks before subsequent analysis. c Peyer's patches were whole-mount immunostained to detect GP2 (green), UEA-1 (red) and f-actin (blue). Closed arrows, GP2 ${ }^{+} \mathrm{M}$ cells with characteristic basolateral pockets; chevrons, GP2-UEA-1+ goblet cells. The positions of the $\mathrm{X}-\mathrm{Z}$ and $\mathrm{Y}-\mathrm{Z}$ projections of the FAE are indicated by the broken lines in the $\mathrm{X}-\mathrm{Y}$ images. Scale bar, $60 \mu \mathrm{m}$. Boxed areas are shown below in higher magnification. Scale bar, $20 \mu \mathrm{m}$. d Morphometric analysis showed that the density of GP2 ${ }^{+} \mathrm{M}$ cells was significantly reduced after prolonged CSF1R blockade, whereas the density of GP2-UEA-1+ goblet cells was significantly increased. Data are mean \pm SD derived from 10 to 13 mice/group, 1 to $9 \mathrm{FAE} /$ mouse from two independent experiments. ${ }^{\star \star \star} P<0.001$, Mann-Whitney $U$-test; ${ }^{\star \star \star \star} P<0.001$, two-tailed unpaired Student's $t$-test. e Morphometric analysis showed that FAE size was unchanged after prolonged CSF1R blockade. Data are mean \pm SEM derived from 10 to 13 mice/group, 1 to $9 \mathrm{FAE} /$ mouse from two independent experiments. NS Mann-Whitney U-test. $\mathbf{f}, \mathbf{g}$ The uptake of particulate antigen (fluorescent beads, green) into the Peyer's patches was significantly impaired in anti-CSF1R-treated mice. Circles highlight individual beads in the SED. Sections counterstained to detect F-actin (blue). Scale bar, $5 \mu \mathrm{m}$. $\mathbf{g}$ The number of beads transcytosed across the FAE was significantly reduced in anti-CSF1R-treated mice. Data represent mean \pm SD from 6 to 12 sections from 4 mice/group. ${ }^{\star \star \star} P<0.001$, Mann-Whitney U-test 
a

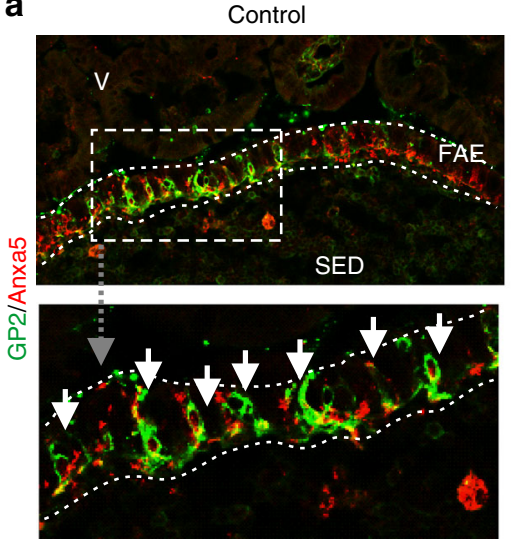

C

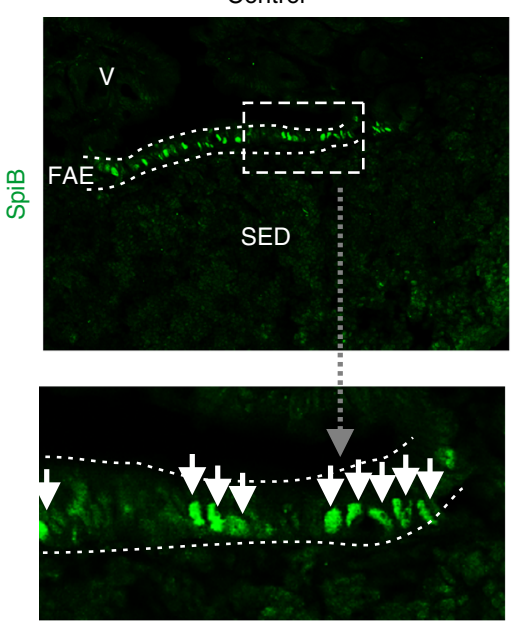

Anti-CSF1R

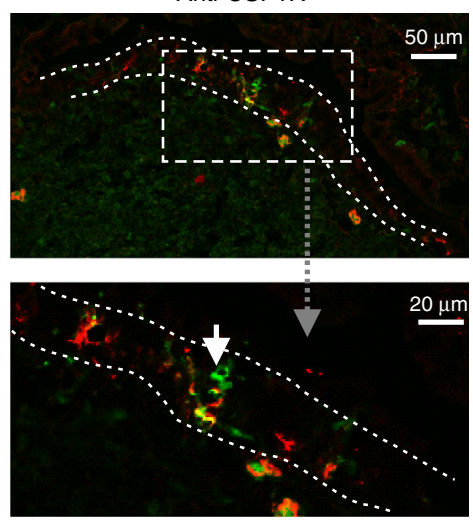

Anti-CSF1R
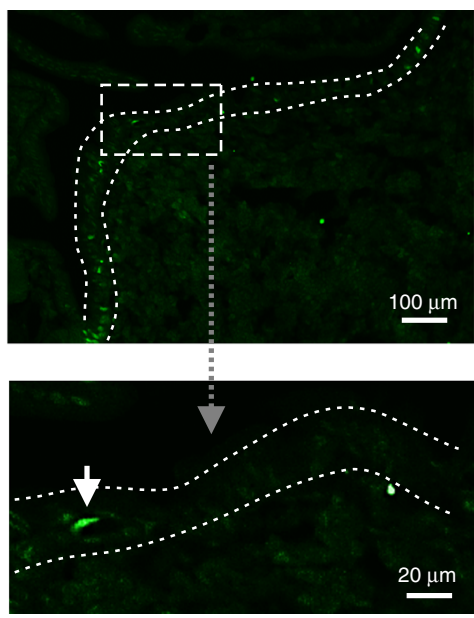

e

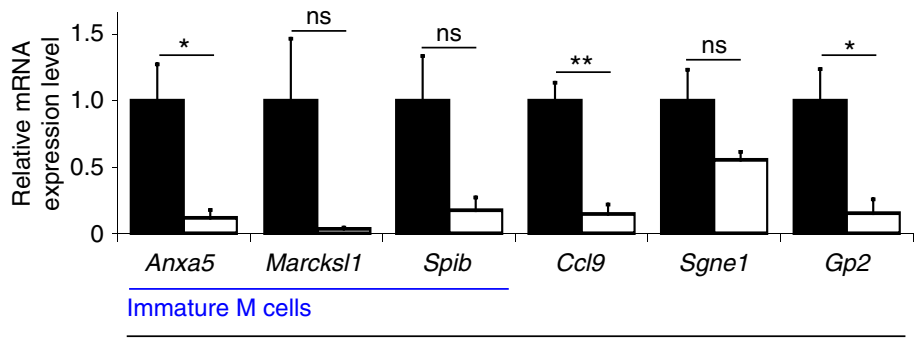

b

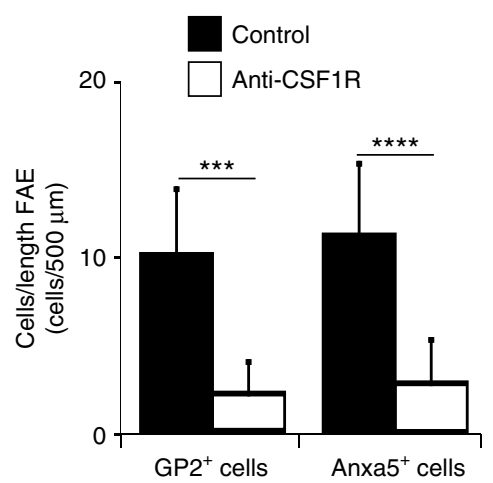

d

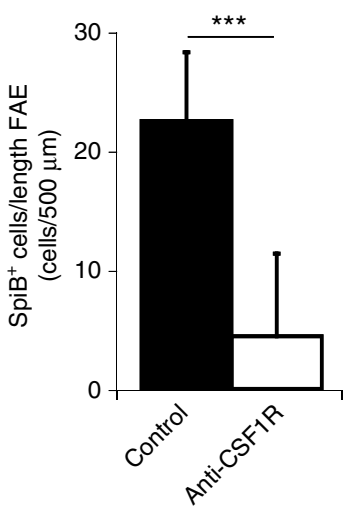

Control

Anti-CSF1R

Fig. 8 Loss of immature and mature M-cell marker expression in Peyer's patches after prolonged CSF1R blockade. Mice were treated with anti-CSF1R mAb or control-lgG (control) for 6 weeks before subsequent analysis. a Detection of Anxa5 (red) and GP2 (green) in the follicle-associated epithelium (FAE) by IHC. Scale bar, upper panels, $50 \mu \mathrm{m}$. Scale bar, lower panel, $20 \mu \mathrm{m}$. b Morphometric analysis of the number of GP2 ${ }^{+}$and Anxa5 $5^{+} \mathrm{M}$ cells in the FAE. SED, subepithelial dome. Data represent mean \pm SD from 1 to 7 FAE from 8 mice/group. ${ }^{\star \star \star}, P<0.001$, two-tailed unpaired Student's $t$-test; ${ }^{\star \star \star \star} P<0.001$, Mann-Whitney $U$-test. c IHC analysis of SpiB expression (green) in the FAE. Dotted line in A \& $C$ shows the FAE boundary. Scale bar, upper panels, $100 \mu$ m. Scale bar, lower panel, $20 \mu \mathrm{m}$. d Morphometric analysis of the number of SpiB ${ }^{+}$cells in the FAE. Data represent mean \pm SD from 6 to 9 FAE from four mice/group. ${ }^{\star \star \star}, P<0.001$, Mann-Whitney U-test. e RT-qPCR analysis shows the expression of immature (Anxa5, Marcks/1, and Spib) and mature (Cc/9, Sgne1, and Gp2) M cell-related genes was reduced in Peyer's patches after prolonged CSF1R blockade. Bars represent mean \pm SEM derived from four mice/ group. ${ }^{\star} P<0.05 ;{ }^{\star \star} P<0.01$, two-tailed unpaired Student's $t$-test. NS not significant

patients with ileal CD display reduced expression of canonical Wnt ligands including Wnt1, Wnt3, and Wnt3a, and Paneth cell function in biopsies from $\mathrm{CD}$ patients can be restored in vitro by PBMC from healthy volunteers ${ }^{59}$. Similarly, macrophages have been shown to promote mucosal repair through activation of Wnt-signaling in a mouse model of inflammatory bowel disease ${ }^{32}$. The corollary of the observed impact of antiCSF1R treatment on intestinal epithelial cell differentiation is that CSF1 treatment has the potential to restore epithelial function following injury, inflammation or chemotherapy, as has already been demonstrated in other organ systems ${ }^{55,56,60,61}$. Efforts to test this hypothesis are ongoing.

\section{Methods}

Mice. The following mouse strains were used where indicated. Male (6-8 weeks old) Csf1r-EGFP reporter "MacGreen" mice ${ }^{12}$ (bred and maintained in-house) were used for all the experiments. Male and female (6-8 weeks old) Vil1-Cre (Tg (Vill-cre)997Gum/J strain; The Jackson Laboratory, Bar Harbor, ME, USA) and 


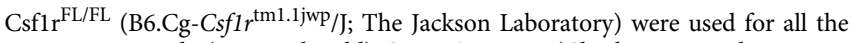
experiments. Male (6-8 weeks old) C57BL/6 J mice (Charles River Laboratories, Tranent, UK) were used for all the experiments. All studies and regulatory licences were approved by the University of Edinburgh's ethics committee and performed under the authority of a UK Home Office Project Licence. All animals were coded before use in the studies described below.
In vivo CSF1R blockade. Mice were injected i.p. with $200 \mu \mathrm{g}$ rat anti-mouse CSF1R monoclonal antibody (mAb; M279; Amgen, Thousand Oaks, CA, USA) three times/week for 6 weeks $^{6,13}$. Rat IgG (I4131; Sigma, Poole, UK) was used as a control. In some experiments a parallel set of mice were treated with anti-CSF1R $\mathrm{mAb}$ for 6 weeks, and then allowed to recover for an additional 8 weeks before analysis.
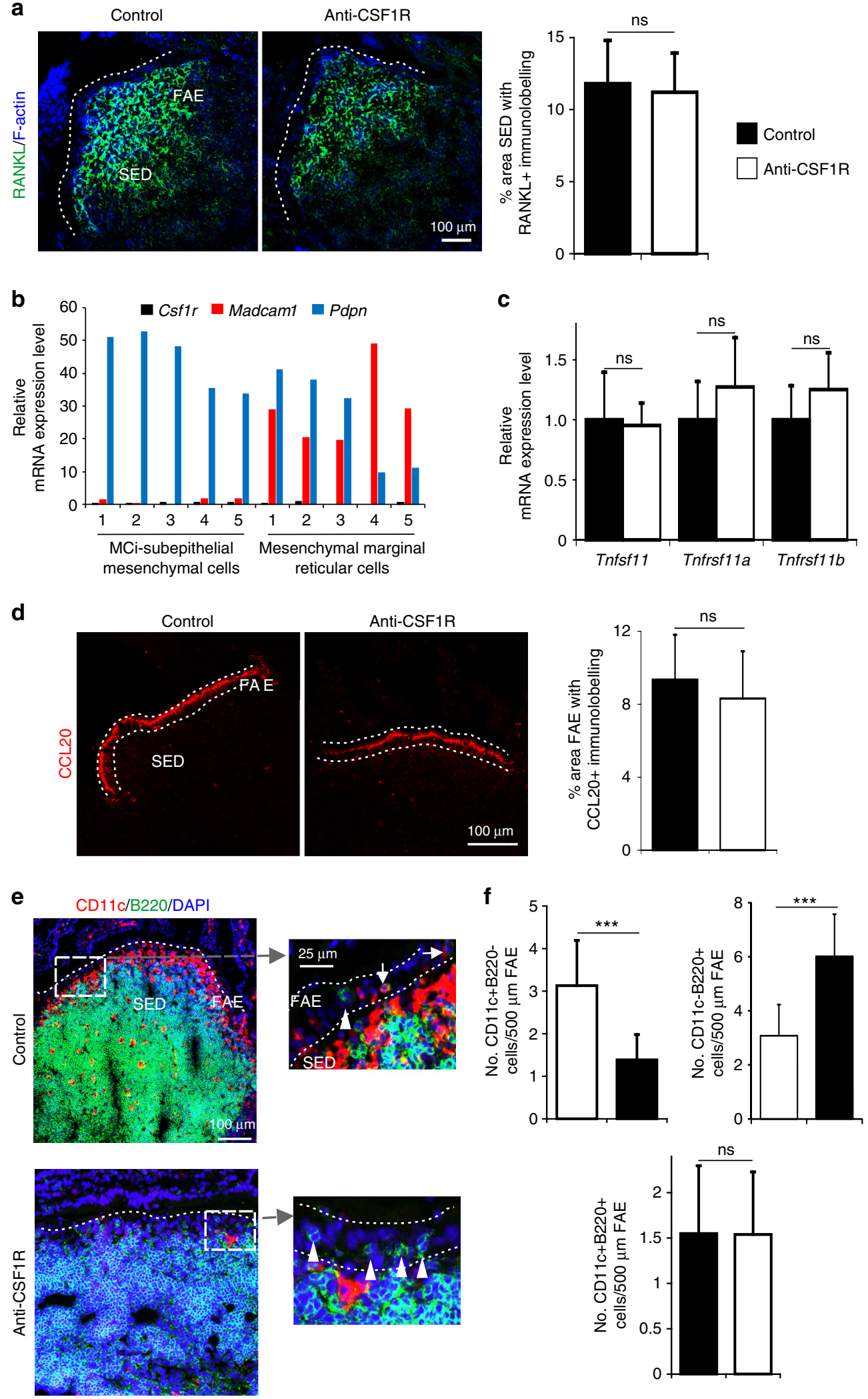
Immunohistochemistry (IHC). Details of the sources, clone numbers and concentrations of all the antibodies used for IHC are provided in Supplementary Table 1. For whole-mount staining, Peyer's patches were fixed with BD Cytofix/ Cytoperm (BD Biosciences, Oxford, UK) and immunostained with rat anti-mouse GP2 mAb (MBL International, Woburn, MA). Tissues were subsequently stained with Alexa Fluor 488-conjugated anti-rat IgG Ab (Invitrogen, Paisley, UK), rhodamine-conjugated Ulex europaeus agglutinin I (UEA-1; Vector Laboratories Inc., Burlingame, CA) and Alexa Fluor 647-conjugated phalloidin (ThermoFisher Scientific, Paisley, UK).

For analysis of tissue sections, intestinal pieces were snap-frozen in liquid nitrogen or fixed in $4 \%$ paraformaldehyde before embedding in optimal cryostat temperature medium (VWR, Leighton Buzzard, UK). Tissue sections $(5 \mu \mathrm{m})$ were immunostained with the primary antibodies listed in Supplementary Table 1. For the detection of lysozyme in intestinal crypts paraformaldehyde-fixed sections were permeabilized with $50 \%$ ethanol for $20 \mathrm{~min}$. before immunostaining with rabbit anti-lysozyme mAb (Abcam, Cambridge, UK). For the detection of SpiB in paraformaldehyde-fixed sections, antigen retrieval was performed with citrate buffer $\left(\mathrm{pH} 7.0,121^{\circ} \mathrm{C}, 5 \mathrm{~min}\right)$ prior to immunostaining with sheep anti-mouse SpiB polyclonal antibody (R\&D Systems, Abingdon, UK). Unless indicated otherwise, following the addition of primary antibody, species-specific secondary antibodies coupled to Alexa Fluor 488 and Alexa Fluor 555 dyes were used (Invitrogen). Sections were counterstained with either Alexa Fluor 647-conjugated phalloidin or DAPI (ThermoFisher), mounted in fluorescent mounting medium (Dako, Ely, UK) and examined using a Zeiss LSM710 confocal microscope (Zeiss, Welwyn Garden City, UK). For quantification of goblet cells in the colon sections were treated with PAS (0.5\% pararosaniline, $1 \%$ sodium metabisulfite).

In vivo assessment of $\boldsymbol{M}$ cell-mediated transcytosis. The uptake of $200 \mathrm{~nm}$ diameter fluoresbrite YG carboxylate microspheres (Polysciences, Eppelheim, Germany) from the gut lumen was assessed as described ${ }^{34}$. Mice ( $n=4$ /group) were anesthetized and $\sim 2 \mathrm{~cm}$ segments of small intestine containing a single Peyer's patch were ligated using nylon filament. The loops were then injected with a $200 \mu \mathrm{l}$ suspension of $200 \mathrm{~nm}$ fluorescent nanoparticles diluted in PBS $\left(\sim 1 \times 10^{11}\right.$ beads/ $\mathrm{ml}$ ). The isolated gut loops were then placed back in the peritoneal cavity. Approximately $1 \mathrm{~h}$ later the injected gut loops were excised, washed in $0.5 \%$ Tween 20/PBS, fixed in $4 \%$ paraformaldehyde/PBS, and embedded in OCT. Frozen sections $(5 \mu \mathrm{m})$ were counterstained with Alexa Fluor 647-conjugated phalloidin and examined using a Nikon Eclipse E400 fluorescence microscope. Data were collected from 10 sections from two Peyer's patches/mouse.

Image analysis. For morphometric analysis, digital microscopy images were analyzed using ImageJ software (http://rsb.info.nih.gov/ij/) as described ${ }^{62}$. Briefly, all images were coded and assessed blindly. Background intensity thresholds were first applied using an ImageJ macro which measures pixel intensity across all immunostained and non-stained areas of the images. The obtained pixel intensity threshold value was then applied in all subsequent analyses. Next, the number of pixels of each color (black, red, green, yellow etc.) were automatically counted and presented as a proportion of the total number of pixels in each area under analysis. In each instance, tissues from 4 to 8 mice/group group were analyzed. In order to analyze the expression of several parameters in each mouse, multiple sections from at least three Peyer's patches were analyzed. In addition at from each mouse between 30 and 120 individual small intestinal and colonic crypts were analyzed.

Crypt isolation and in vitro enteroid cultivation. Intestinal crypts were dissociated from mouse small intestine using Gentle Cell Dissociation Reagent (Stemcell Tech, Cambridge, UK). Crypts were subsequently used in mRNA expression analyses or for establishing enteroids by cultivation in Matrigel (BD
Bioscience, Oxford, UK) and Intesticult medium (Stemcell Technologies, Cambridge, UK) ${ }^{63,64}$. Briefly, crypts were re-suspended in Intesticult medium at $2 \times 10^{3}$ crypts $/ \mathrm{ml}$ and mixed 1:1 with Matrigel. Next, $50 \mu \mathrm{l}$ Matrigel plugs were plated in pre-warmed 24-well plates and allowed to settle, before addition of $600 \mu \mathrm{l}$ of prewarmed Intesticult medium and subsequently cultured at $37^{\circ} \mathrm{C}$ in a $5 \% \mathrm{CO}_{2}$ atmosphere. Fresh medium was replaced every $2 \mathrm{~d}$ of cultivation. Where indicated, some wells were treated with either $10 \mu \mathrm{g} / \mathrm{ml}$ anti-CSF1R mAb (or $10 \mu \mathrm{g} / \mathrm{ml}$ rat IgG

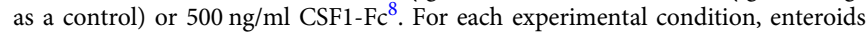
were cultivated in triplicate and repeated on three independent occasions. After $5 \mathrm{~d}$ of cultivation the enteroids were either harvested for mRNA expression analysis as described ${ }^{64}$, or passaged as described ${ }^{63}$

To passage the enteroids, ice cold DMEM F/12 medium (Life Technologies, Paisley, UK) was added to the wells and the enteroids vigorously pipetted to disrupt the Matrigel plug. The enteroids were then allowed to settle by gravity, the supernatant discarded and then washed 3 more times in ice cold DMEM F/12. Crypts were physically disassociated from the main enteroids bodies by vigorous pipetting through a flame-polished glass Pasteur pipette with the tip stretched to reduce the aperture. The dissociated crypts were then allowed to settle by gravity, counted using a haemocytometer, re-suspended in Intesticult medium and enteroid cultures established as above.

For mRNA extraction, the Matrigel plug containing the enteroids was incubated in $500 \mu \mathrm{l}$ of Corning Cell Recovery Solution (Corning, Wycombe, UK) for $1 \mathrm{~h}$ at $4^{\circ} \mathrm{C}$. Once all the Matrigel was depolymerized, the cell pellet was then homogenized in the RLT buffer provided by the DNeasy Blood and Tissue Kit (Qiagen, Manchester, UK). Total RNA was then extracted using the DNeasy Blood and Tissue Kit according to the manufacturer's instructions.

Real-time quantitative PCR (RT-qPCR) analysis of mRNA expression. Total RNA was isolated from tissues and crypts using RNA-Bee (AMS Biotechnology, Oxfordshire, UK) followed by treatment with DNase I (Ambion, Warrington, UK). First strand cDNA synthesis was performed using $1 \mu \mathrm{g}$ of total RNA and the First Strand cDNA Synthesis kit (GE Healthcare, Bucks, UK) as described by the manufacturer. PCR was performed using the Platinum-SYBR Green $\mathrm{QPCR}$ SuperMix-UDG kit (Invitrogen) and the Stratagene Mx3000P real-time qPCR system (Stratagene, CA, USA). The qPCR primers (Supplementary Table 2) were designed using Primer3 software ${ }^{65}$. The cycle threshold values were determined using MxPro software (Stratagene) and normalized relative to Gapdh.

RNA in situ hybridizations. Paraformaldehyde-fixed intestine sections $(5 \mu \mathrm{m}$ in thickness) were processed for RNA in situ hybridization detection using RNAscope $2.5 \mathrm{HD}$ single (red) or duplex (chromogenic) detection kits according to the manufacturer's instructions (Advanced Cell Diagnostics, Hayward, CA, USA). Target retrieval and protease digestions were performed for 15 and 30 mins, respectively. The RNAscope probes used are shown in Supplementary Table 3. Positive and negative control probes were used for each experiment according to the manufacturer's instructions. For analysis of Bmil expression the duration of amplification step 5 was increased to $1 \mathrm{~h}$.

Statistical analyses. Details of all group/sample sizes and experimental repeats are provided in the figure legends. Unless indicated otherwise, all data are presented as mean \pm SD and differences between groups analyzed by a two-sided Student's $t$ test. In instances where there was evidence of non-normality (identified by the Kolmogorov-Smirnov test), data were analyzed by a Mann-Whitney $U$-test. Values of $P<0.05$ were accepted as significant.

Data availability. The authors declare that all data supporting the findings in this study are available within the article and its Supplementary Information files or

Fig. 9 Prolonged CSF1R blockade does not affect RANKL or CCL2O expression in Peyer's patches. a IHC analysis shows no observable difference in the expression or distribution of RANKL on subepithelial dome (SED) stromal cells after anti-CSF1R mAb treatment. Scale bar $100 \mu$ m. Morphometric analysis (histogram, right) confirmed the magnitude of the RANKL-specific immunostaining in the SED of Peyer's patches from each group of mice was similar. Data represent mean \pm SD from 3 to 4 mice/group and 1 to 3 SED regions/mouse. NS Mann-Whitney U-test. b Expression of Csf1r (black), Madcam1 (red) and Pdpn (blue) in individual mRNA sequencing datasets of mouse "M cell-inducing-" (MCi)-subepithelial mesenchymal cells and mesenchymal marginal reticular cells ${ }^{37}$. c RT-qPCR analysis shows no significant difference in the expression of Tnfsf11 (RANKL), Tnfrsf11a (RANK), or Tnfrsf11b (OPG) mRNA in Peyer's patches following anti-CSF1R treatment. Bars represent mean \pm SEM derived from four mice/group. NS not significant, two-tailed unpaired Student's $t$-test. $\mathbf{d} I \mathrm{HC}$ analysis shows no observable difference in the expression of CCL2O in the follicle-associated epithelium (FAE) after anti-CSF1R mAb treatment. Scale bar, $100 \mu \mathrm{m}$. Morphometric analysis (histogram, right) confirmed the magnitude of the CCL20-specific immunostaining in Peyer's patches from each group of mice was similar. Data represent mean \pm SD from 3 to 9 FAE from four mice/group. NS two-tailed unpaired Student's $t$-test. e IHC

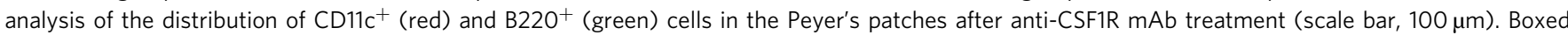
areas are shown adjacently in higher magnification (scale bar, $25 \mu \mathrm{m}$ ). Arrows indicate CD11c ${ }^{+} \mathrm{B} 22 \mathrm{O}^{+}$" $\mathrm{M}$ cell-inducing" B cells. Arrow-heads indicate $\mathrm{CD}_{11} \mathrm{c}^{-} \mathrm{B} 22 \mathrm{O}^{+} \mathrm{B}$ cells. Sections counterstained with DAPI to detect nuclei (blue). f After anti-CSF1R treatment morphometric analysis showed that the number of $\mathrm{CD}_{11 \mathrm{c}^{+}} \mathrm{B}_{22 \mathrm{O}^{-}}$mononuclear phagocytes in the FAE was significantly decreased (left-hand panel), the number of $\mathrm{CD} 11 \mathrm{c}^{-} \mathrm{B} 22 \mathrm{O}^{+} \mathrm{B}$ cells was increased (right-hand panel), but the number of $\mathrm{CD} 11 \mathrm{c}^{+} \mathrm{B} 22 \mathrm{O}^{+}$"M cell-inducing" B cells was unchanged (lower panel). Dotted line indicates the FAE boundary. Data represent mean \pm SD from 1-11 FAE from four mice/group. ${ }^{\star \star \star} P<0.001$, two-tailed unpaired Student's $t$-test. NS not significant 
from the corresponding author on reasonable request. The following published mRNA expression datasets were also analyzed which are publicly available in the Gene Expression Omnibus (https://www.ncbi.nlm.nih.gov/gds) via the following accession codes: GSE53297; GSE93320; GSE100393. Deep CAGE sequence data from the FANTOM consortium ${ }^{25}$ are available via the following URL: http:// fantom.gsc.riken.jp/.

Received: 4 May 2016 Accepted: 2 March 2018

Published online: 28 March 2018

\section{References}

1. Sato, T. et al. Single Lgr5+ stem cells build crypt-villus structures in vitro without a mesenchymal niche. Nature 459, 262-265 (2009).

2. Sato, T. et al. Paneth cells constitute the niche for Lgr5 stem cells in intestinal crypts. Nature 469, 415-418 (2011).

3. Bain, C. C. et al. Constant replenishment from circulating monocytes maintains the macrophage pool in the intestine of adult. Nat. Immunol. 15, 929-937 (2014).

4. Zigmond, E. \& Jung, S. Intestinal macrophages: well educated exceptions from the rule. Trends Immunol. 34, 162-168 (2013).

5. Dai, X. M. et al. Targeted disruption of the mouse colony-stimulating factor 1 receptor gene results in osteopetrosis, mononuclear phagocyte deficiency, increased primitive progenitor cell frequencies, and reproductive defects. Blood 99, 111-120 (2002).

6. MacDonald, K. P. et al. An antibody against the colony-stimulating factor 1 receptor depletes the resident subset of monocytes and tissue- and tumourassociated macrophages but does not inhibit inflammation. Blood 116, 3955-3963 (2010).

7. Hume, D. A., Pavli, P., Donahue, R. E. \& Fidler, I. J. The effect of human recombinant macrophage colony-stimulating factor (CSF-1) on the murine mononuclear phagocyte system in vivo. J. Immunol. 141, 3405-3409 (1988).

8. Gow, D. et al. Characterisation of a novel Fc conjugate of Macrophage Colony-Stimulating Factor (CSF1). Mol. Ther. 22, 1580-1592 (2014).

9. Akcora, D. et al. The CSF-1 receptor fashions the intestinal stem cell niche. Stem Cell Res. 10, 203-212 (2013).

10. Huynh, D. et al. Colony stimulating factor-1 dependence of Paneth cell development in the mouse small intestine. Gastroenterology 137, 136-144 (2009).

11. Huynh, D. et al. CSF-1 receptor-dependent colon development, homeostasis and inflammatory stress response. PLoS ONE 8, e56951 (2013).

12. Sasmono, R. T. et al. A macrophage colony-stimulating factor receptor-green fluorescent protein transgene is expressed throughout the mononuclear phagocyte system of the mouse. Blood 101, 1155-1163 (2003).

13. Sauter, K. A. et al. Pleiotropic effects of extended blockade of CSF1R signalling in adult mice. J. Leukoc. Biol. 96, 265-274 (2014).

14. Pollard, J. W. Trophic macrophages in development and disease. Nat. Rev. Immunol. 9, 259-270 (2009).

15. Muller, P. A. et al. Crosstalk between muscularis macrophages and enteric neurones regulates gastrointestinal motility. Cell 158, 300-313 (2014).

16. Gabanyi, I. et al. Neuro-immune interactions drive tissue programming in intestinal macrophages. Cell 164, 378-391 (2016).

17. de Lau, W. et al. Peyer's patch M cells derive from Lgr5+ stem cells, require SpiB and are induced by RankL in cultured 'organoids'. Mol. Cell. Biol. 32, 3639-3647 (2012).

18. Mabbott, N. A., Donaldson, D. S., Ohno, H., Williams, I. R. \& Mahajan, A Microfold (M) cells: important immunosurveillance posts in the intestinal epithelium. Mucosal Immunol. 6, 666-677 (2013).

19. Hase, K. et al. Uptake through glycoprotein 2 of $\mathrm{FimH}^{+}$bacteria by $\mathrm{M}$ cells initiates mucosal immune responses. Nature 462, 226-231 (2009).

20. Kanaya, T. et al. The Ets transcription factor Spi-B is essential for the differentiation of intestinal microfold cells. Nat. Immunol. 13, 729-736 (2012).

21. Shima, $H$. et al. A novel vaccine targeting Peyer's patch $M$ cells induces protective antigen-specific IgA responses. Int. Immunol. 26, 619-625 (2014).

22. Banaei-Bouchareb, L. et al. Insulin cell mass is altered in $C s f 1^{\circ p} / C s f 1^{o p}$ macrophage-deficient mice. J. Leukoc. Biol. 76, 359-367 (2004).

23. Sasmono, R. T. et al. Mouse neutrophilic granulocytes express mRNA encoding the macrophage colony-stimulating factor receptor (CSF-1R) as well as many other macrophage-specific transcripts and can transdifferentiate into macrophages in vitro in response to CSF-1. J. Leukoc. Biol. 82, 111-123 (2007).

24. Mooney, J. E. et al. Cellular plasticity of inflammatory myeloid cells in the peritoneal foreign body response. Am. J. Pathol. 176, 369-380 (2010).

25. FANTOM Consortium and the RIKEN PMI and CLST (DGT), Forrest, A. R. et al. A promoter-level mammalian expression atlas. Nature 507, 462-470 (2014).
26. Grun, G. et al. Single-cell messenger RNA sequencing reveals rare intestinal cell types. Nature 525, 251-255 (2015).

27. Middendorp, S. et al. Adult stem cells in the small intestine are intrinsically programmed with their location-specific function. Stem Cells 32, 1083-1091 (2014).

28. Tian, H. et al. A reserve stem cell population in small intestine renders Lgr5positive cells dispensable. Nature 478, 255-259 (2011).

29. Yan, K. S. et al. The intestinal stem cell markers Bmil and Lgr5 identify two functionally distinct populations. Proc. Natl Acad. Sci. USA 109, 466-471 (2012).

30. Sangiorgi, E. \& Capecchi, M. R. Bmil is expressed in vivo in intestinal stem cells. Nat. Genet. 40, 915-920 (2008).

31. Sasaki, N. et al. Reg4 ${ }^{+}$deep crypt secretory cells function as epithelial niche for $\mathrm{Lgr}^{+}{ }^{+}$stem cells in colon. Proc. Natl Acad. Sci. USA 113, E5399-E5407 (2016).

32. Cosin-Roger, J. et al. The activation of Wnt signaling by a STAT6-dependent macrophage phenotype promotes mucosal repair in murine IBD. Mucosal Immunol. 9, 986-998 (2015).

33. Saha, S. et al. Macrophage-derived extracellular vesicle-packaged WNTs rescue intetinal stem cells and enhance survival after radiation injury. Nat. Commun. 7, 13096 (2016).

34. Kobayashi, A. et al. The functional maturation of $\mathrm{M}$ cells is dramatically reduced in the Peyer's patches of aged mice. Mucosal Immunol. 6, 1027-1037 (2013).

35. Knoop, K. A. et al. RANKL is necessary and sufficient to initiate development of antigen-sampling $\mathrm{M}$ cells in the intestinal epithelium. J. Immunol. 183, 5738-5747 (2009)

36. Wittrant, Y., Gorin, Y., Mohan, S., Wager, B. \& Abboud-Werner, S. L. Colonystimulating factor-1 (CSF-1) directly inhibits receptor activator of nuclear factor-kB ligand (RANKL) expression by osteoclasts. Endocrinology 150, 4977-4988 (2009)

37. Nagashima, K. et al. Identification of subepithelial mesenchymal cells that induce IgA and diversify gut microbiota. Nat. Immunol. 18, 675-682 (2017).

38. Ebisawa, M. et al. CCR6hiCD11cint B cells promote M-cell differentiation in Peyer's patch. Int. Immunol. 23, 261-269 (2011).

39. Farin, H. F. et al. Visualization of a short-range Wnt gradient in the intestinal stem-cell niche. Nature 530, 340-343 (2016).

40. van Es, J. H. et al. Wnt signalling induces maturation of Paneth cells in intestinal crypts. Nat. Cell Biol. 7, 381-386 (2005).

41. Kim, T.-H., Escudero, S. \& Shivdasani, R. A. Intact functin of Lgr5 receptorexpressing intestinal stem cells in the absence of Paneth cells. Proc. Natl Acad. Sci. USA 109, 3932-3937 (2012).

42. San Roman, A. K., Jayewickreme, C. D., Murtaugh, L. C. \& Shivdasani, R. A Wnt secretion from epithelial cells and subepithelial myofibroblasts is not required in the mouse intestinal stem cell niche in vivo. Stem Cell Rep. 2, 127-134 (2014).

43. Farin, H. F., van Es, J. H. \& Clevers, H. Redundant sources of Wnt regulate intestinal stem cells and promote formation of Paneth cells. Gastroenterology 143, 1518-1529 (2012).

44. Nakamura, K., Sakuragi, N., Takakuwa, A. \& Ayabe, T. Paneth cell $\alpha$-defensins and enteric microbiota in health and disease. Biosci. Micro. Food Health $\mathbf{3 5}$ $57-67$ (2016)

45. Hayase, E. et al. R-spondin 1 expands Paneth cells and prevents dysbiosis induced by graft-versus-host disease. J. Exp. Med. 214, 3507-3518 (2017).

46. Riba, A. et al. Paneth cell defects induce microbiota dysbiosis in mice and promote visceral hypersensitivity. Gastroenterology 153, 1594-1606 (2017).

47. Chow, A. et al. Bone marrow $\mathrm{CD} 169^{+}$macrophages promote the retention of hematopoietic stem and progenitor cells in the mesenchymal stem cell niche. J. Exp. Med. 208, 261-271 (2011).

48. Chow, A. et al. $\mathrm{CD} 169^{+}$macrophages provide a niche promoting erythropoiesis under homeostasis and stress. Nat. Immunol. 19, 429-436 (2013).

49. Chang, M. K. et al. Osteal tissue macrophages are intercalated throughout human and mouse bone lining tissues and regulate osteoblast function in vitro and in vivo. J. Immunol. 181, 1232-1244 (2008).

50. Mulley, A. et al. Myeloid Wnt ligands are required for normal development of the dermal lymphatic vasculature. PLoS ONE 12, e0181549 (2017).

51. Date, S. \& Sato, T. Mini-gut organoids: reconstitution of the stem cell niche. Annu. Rev. Cell. Dev. Biol. 31, 269-289 (2015).

52. Ryan, G. R. et al. Rescue of the colony-stimulating factor 1 (CSF-1)nullizygous mouse $\left(\mathrm{Cs} f 1^{o p} / \mathrm{Cs} f 1^{o p}\right)$ phenotype with a CSF-1 transgene and identification of sites of local CSF-1 synthesis. Blood 98, 74-84 (2001).

53. Hernandez, L. et al. The EGF/CSF-1 paracrine invasion loop can be triggered by heregulin beta1 and CXCL12. Cancer Res. 69, 3221-3227 (2009).

54. De Lau, W. et al. Lgr5 homologues associate with Wnt receptors and mediate R-spondin signalling. Nature 476, 293-297 (2011).

55. Menke, J. et al. CSF-1 signals directly to renal tubular epithelial cells to mediate repair in mice. J. Clin. Invest. 119, 2330-2342 (2009). 
56. Alikhan, M. A. et al. Colony-stimulating factor-1 promotes kidney growth and repair via alteration of macrophage responses. Am. J. Pathol. 179, 1243-1256 (2011).

57. Heuberger, J. et al. Shp2/MAPK signaling controls goblet/paneth cell fate decision in the intestine. Proc. Natl Acad. Sci. USA 111, 3472-3477 (2014).

58. Hume, D. A. \& MacDonald, K. P. Therapeutic applications of macrophage colony-stimulating factor-1 (CSF-1) and antagonists of CSF-1 receptor (CSF1R) signaling. Blood 119, 1810-1820 (2012).

59. Courth, L. F. et al. Crohn's disease-derived monocytes fail to induce Paneth cell defensins. Proc. Natl Acad. Sci. USA 112, 14000-14005 (2015).

60. Alexander, K. A. et al. Osteal macrophages promote in vivo intramembranous bone healing in a mouse tibeal injury model. J. Bone Miner. Res. 2011, 1517-1532 (2011).

61. Stutchfield, B. M. et al. CSF1 restores innate immunity after liver injury in mice and serum levels indicate outcomes of patients with acute liver failure. Gastroenterology 149, 1896-1909 (2015).

62. Inman, C. F. et al. Validation of computer-assisted, pixel-based analysis of multiple-colour immunofluorescence histology. J. Immunol. Met 302, 156-167 (2005).

63. Sato, T. \& Clevers, H. Primary mouse small intestinal epithelial cell cultures. Methods Mol. Biol. 945, 319-328 (2013).

64. Rios, D. et al. Antigen sampling by intestinal $M$ cells is the principal pathway initiating mucosal IgA production to commensal enteric bacteria. Mucosal Immunol. 9, 907-916 (2016).

65. Untergasser, A. et al. Primer3Plus, an enhanced web interface to Primer3. Nucleic Acids Res. 35, W71-W74 (2007).

\section{Acknowledgements}

We thank Bob Fleming and Barry Bradford (Univ. Edinburgh) for excellent technical support. This study was supported by supported project (BB/J014672/1, BB/M024288/1 and BB/K021257/1), Institute Strategic Programme Grant (BBS/E/D/20231762; BBS/E/ $\mathrm{D} / 10002071)$ and an EASTBIO doctoral training programme studentship (BB/J01446X/ 1) funding from the Biotechnology and Biological Sciences Research Council and project grant funding from the Medical Research Council (MR/M019969/1).

\section{Author contributions}

D.A.H. and N.A.M. conceived the study, A.S., D.S.D., K.A.S., C.P., D.A.H., and N.A.M. designed the study; A.S., D.S.D., K.A.S., and C.P. acquired these data; all authors interpreted these data and contributed to the final version of this report.

\section{Additional information}

Supplementary Information accompanies this paper at https://doi.org/10.1038/s41467018-03638-6.

Competing interests: The authors declare no competing interests.

Reprints and permission information is available online at http://npg.nature.com/ reprintsandpermissions/

Publisher's note: Springer Nature remains neutral with regard to jurisdictional claims in published maps and institutional affiliations.

\begin{abstract}
(c) (i) Open Access This article is licensed under a Creative Commons Attribution 4.0 International License, which permits use, sharing, adaptation, distribution and reproduction in any medium or format, as long as you give appropriate credit to the original author(s) and the source, provide a link to the Creative Commons license, and indicate if changes were made. The images or other third party material in this article are included in the article's Creative Commons license, unless indicated otherwise in a credit line to the material. If material is not included in the article's Creative Commons license and your intended use is not permitted by statutory regulation or exceeds the permitted use, you will need to obtain permission directly from the copyright holder. To view a copy of this license, visit http://creativecommons.org/ licenses/by/4.0/.
\end{abstract}

(C) The Author(s) 2018 